\title{
Energy correlations in random transverse field Ising spin chains
}

\author{
Gil Refael and Daniel S. Fisher \\ Department of Physics, Harvard University, Cambridge, Massachusetts 02138, USA \\ (Received 8 August 2003; revised manuscript received 28 April 2004; published 17 August 2004)
}

\begin{abstract}
The end-to-end energy-energy correlations of random transverse field quantum Ising spin chains are computed using a generalization of an asymptotically exact real space renormalization group (RG) previously introduced. Away from the critical point, the average energy-energy correlations decay exponentially with a correlation length that is the same as that of the spin-spin correlations. The typical correlations, however, decay exponentially with a characteristic length, proportional to the square root of the primary correlation length. At the quantum critical point, the average correlations decay subexponentially as $\overline{C_{L}} \sim e^{- \text {const } L^{1 / 3}}$, whereas the typical correlations decay faster, as $\sim e^{-K \sqrt{L}}$, with $K$ a random variable with a universal distribution. The critical energy-energy correlations behave very similarly to the smallest gap, computed previously; this is explained in terms of the RG flow and the excitation structure of the chain. In order to obtain the energy correlations, an extension of the previously used methods was needed; here, this was carried out via RG transformations that involve a sequence of unitary transformations.
\end{abstract}

DOI: $10.1103 /$ PhysRevB.70.064409

PACS number(s): 75.10.Jm, 64.60.Ak

\section{INTRODUCTION}

The random transverse field Ising model [Eq. (1)] is the prototypical model of a quantum system with quenched randomness. In this model, a ferromagnetic Ising interaction in the $z$ direction competes with a magnetic field in the $x$ direction. Because the Hamiltonian contains both the $x$ and $z$ spin operators that do not commute with each other, and thus involves quantum fluctuations, it is sometimes referred to as the quantum Ising model. Reference 1 gives a thorough review of the pure transverse field Ising model. The random transverse field Ising model undergoes a unusual quantum phase transition at zero temperature that is controlled by an infinite randomness fixed point. ${ }^{2,3}$ As a consequence, over a wide range of parameters, unusual low-temperature behavior is exhibited. In one dimension, many of the low-energy properties have been found exactly, initially by $\mathrm{McCoy}$ and $\mathrm{Wu}^{4,5}$ and by various authors recently., $3,6,7$ The behavior in higher dimensions has been shown to be in the same general class as the one-dimensional system. ${ }^{8,9}$

In addition to the theoretical interest of the transverse field Ising model, models with similar behavior have been argued to be relevant for the low-temperature properties of heavy fermion materials, with randomness and proximity to a quantum critical point playing key roles in producing nonfermi-liquid behavior. ${ }^{10}$

Much is already known about the random transverse field Ising chain. Previous calculations have obtained the average magnetization and $\sigma^{2}$ correlations. ${ }^{3}$ But, as is often the case in random systems, even in large samples some physical quantities do not converge to a single value, but have a nontrivial distribution. In addition, the resulting distributions of physical quantities are often such that the average value of the quantity differs greatly from the typical value (defined, for example, as the median of the distribution). ${ }^{2,3,11-13} \mathrm{Be}-$ cause of the ubiquity of this type of behavior, it is clear that knowledge about the probability distributions of physical quantities is crucial for a full understanding of random systems. In Ref. 6, the distribution of the order-parameter $\left(\hat{\sigma}^{z}\right)$ end-to-end correlation function is calculated, providing a sharp illustration of this point. The distribution of the orderparameter correlations becomes a delta function (centered on its typical value) in the limit of infinite length; nevertheless, this is misleading: the average correlations even in the infinite length limit are much stronger than the typical correlations that are obtained for almost all samples.

In this paper, we study the end-to-end Ising exchange energy $\left(\hat{\sigma}^{z} \hat{\sigma}^{z}\right)$ and transverse spin $\left(\hat{\sigma}^{x}\right)$ correlations of long, but finite, random transverse field Ising chains. The transverse spin correlations are as important to understand as the orderparameter correlations (i.e., $\hat{\sigma}^{z}$ correlations). Experimentally, the two correlation functions differ only in the polarization of the neutron beam, or, alternatively, the direction of a probing magnetic field. Furthermore, mechanical distortions of the chain couple to its magnetic degrees of freedom through the Ising interaction, so that spin Peierls and other magnetoelastic effects are controlled by $\hat{\sigma}^{z} \hat{\sigma}^{z}$ correlations. The behavior of the Ising energy and transverse magnetization correlations are, as one would expect, significantly different from those of the order parameter; indeed, it is not at all clear whether these correlations should exhibit the disparity between their average and typical values that characterize the order-parameter correlations. Our analysis of the distributions of these quantities shows that such disparity indeed exists, but in a more subtle way.

Computing the distributions of the Ising energy and transverse spin correlations introduces new difficulties that compel us to further develop the general renormalization group (RG) structure that was previously introduced. ${ }^{3,6}$ The formulation presented here should be useful for other random quantum systems, in addition to random quantum Ising models. In particular, it should enable the study of timedependent correlation functions, needed for the understanding of the dynamics of random quantum systems.

The organization of this paper is as follows: in the remainder of this section, we review some aspects of the random Ising model and introduce the quantities of interest. In Sec. II we develop and apply a unitary-transformation ap- 


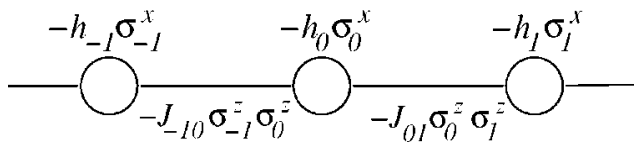

FIG. 1. The Hamiltonian of the transverse field model. Each site is a spin-1/2 that interacts via Ising exchange with its nearest neighbors and can be flipped by the local $x$-magnetic field.

proach to the real space RG. In Secs. III and IV Laplace transforms of the energy and transverse spin correlations are derived and used in Sec. V to obtain the average and typical correlations, and more general information about the distributions. Finally, Sec. VI presents conclusions. Some technical details are relegated to an appendix.

\section{A. Random transverse field Ising model}

The Hamiltonian of the random quantum Ising model is

$$
\mathcal{H}=-\sum_{i}\left(J_{i+1} \hat{\sigma}_{i}^{z} \hat{\sigma}_{i+1}^{z}+h_{i} \hat{\sigma}_{i}^{x}\right)
$$

with each site having two states, $\hat{\sigma}^{z}= \pm 1$, with quantum fluctuations between them caused by the transverse, $\hat{\sigma}^{x}$, fields. The system is illustrated in Fig. 1. Note that there are no magnetic fields in the $z$ direction, so that there is a global symmetry of inversion about the $x y$ spin plane. The presence of $z$ fields would break this symmetry and change the lowenergy physics radically.

The quantum Ising model exhibits a quantum phase transition in its ground state when the nearest-neighbor interaction and the transverse field are of comparable strength. In a nonrandom model this occurs when $J=h$. In a random system, where the $J$ 's and the $h$ 's are drawn independently from some distributions, the transition occurs when $\overline{\log h}=\overline{\log J}$, where the overbars denote averaging over the randomness. A convenient parametrization of the proximity to the transition is

$$
\delta \equiv \frac{\overline{\log h}-\overline{\log J}}{\operatorname{var}(\log h)+\operatorname{var}(\log J)},
$$

with $\delta$ positive in the disordered phase.

\section{B. Real space RG}

A powerful route to analytic information on this system is a real space - or energy space- $\mathrm{RG}$ method that is a generalization developed by one of us, ${ }^{3}$ of an RG introduced by Ma, Dasgupta, and Hu. ${ }^{14,15}$ The real space RG is carried out by decimating the term in the Hamiltonian-a site $\left(h \hat{\sigma}^{x}\right)$, or a bond $\left(J \hat{\sigma}^{z} \hat{\sigma}^{z}\right)$-with the strongest interaction; second-order perturbation theory results in new effective couplings. In the case of decimating a bond, a cluster forms with a renormalized transverse field; in the case of decimating a spin, a new Ising interaction that couples its two neighbors forms. The Hamiltonian preserves its form, with effective bonds coupling spin clusters, and the energy scale-the maximum remaining coupling - reduced. The effective bond strengths and lengths, as well as the effective transverse fields on the clusters of spins and their moments can be computed.

As the energy scale is systematically reduced, the distributions of the effective couplings become very broad for small $\delta$. Concomitantly, the averages of many quantities in the ground state are determined by rare tails of their distributions. An example of this behavior, which was mentioned above in Sec. I, is given by the order-parameter correlations, $C^{z z}(n, n+r)=\left\langle\hat{\sigma}_{n}^{z} \hat{\sigma}_{n+r}^{z}\right\rangle$. Typically, the two sites $n$ and $n+r$ have correlations that decay exponentially with $r$. Nevertheless, at large separations, $r$, the average order-parameter correlations,

$$
\overline{C^{z z}(n, n+r)} \equiv \overline{\left\langle\hat{\sigma}_{n}^{z} \hat{\sigma}_{n+r}^{z}\right\rangle},
$$

are dominated by the rare realizations for which the two spins, $n$ and $n+r$, are almost perfectly correlated with each other in the ground state, i.e., they belong to the same cluster. In the RG picture, this event happens when the two spins in question are not decimated until they join together into the same spin cluster. Although the probability for this to happen vanishes in the limit $r \rightarrow \infty$, and therefore constitutes a rare tail of the correlations' distribution, it vanishes less rapidly than the typical correlations, and hence, dominates the average. $^{3}$

\section{Logarithmic energy scaling}

Many properties of the random quantum Ising model can be understood in terms of the scaling behavior of the cluster sizes, bond lengths, and coupling strengths with the energy scale and the deviation from criticality, $\delta$. At the critical point, the distributions of $h_{i}$ and $J_{i+1}$ become infinitely broad as the energy scale, $\Omega$ approaches zero; the random quantum critical point is thus an infinite randomness fixed point. ${ }^{2,8,16}$ At this fixed point, the distribution of cluster and bond lengths, the logarithms of the interactions in units of $\Omega$,

$$
\begin{aligned}
& \zeta \equiv \log \frac{\Omega}{J}, \\
& \beta \equiv \log \frac{\Omega}{h},
\end{aligned}
$$

and the deviation from criticality, $\delta$, all scale with the logarithm of the energy scale,

$$
\Gamma \equiv \log \frac{\Omega_{I}}{\Omega} .
$$

Here, $\Omega_{I}$ is the initial energy scale set by the strongest couplings, and $\Omega$ is the magnitude of the largest remaining couplings after the stronger ones have been decimated. The scaling can equivalently be given in terms of a length scale, $\ell$-for example, the length of an effective bond - the scaling of $\log$ energies at fixed $\ell$ is of the form

$$
\begin{gathered}
\zeta=z \sqrt{l}, \\
\Gamma=\gamma \sqrt{l},
\end{gathered}
$$

where $\gamma$ and $z$ are scale invariant random variables. 
Various basic results follow directly from this scaling. In particular, the linear number density of remaining spin clusters at scale $\Gamma$ is

$$
n \approx \frac{n_{0}}{\Gamma^{2}}
$$

with $n_{0}$ a nonuniversal prefactor inversely proportional to the original bond lengths.

An example of the scaling of log-energies with length is the gap, $E_{1}-E_{0}$, between the ground state and the first excited state of long finite chains: Analytic and numerical results show that near the critical point the logarithm of the gap is broadly distributed on the scale $\sqrt{L}$. Indeed, the distribution of $-\ln \left(E_{1}-E_{0}\right) / \sqrt{L}$ attains a universal scaling form in the large $L$, and small $\delta$ limit. $^{6}$ From the RG structure, this can be seen by noting that the gap is the energy scale of the chain when it has only one remaining cluster-and thus, only one unfrozen degree of freedom. Therefore, the gap is approximately $\Omega_{L} \sim e^{-\Gamma_{L}}$, with $\Gamma_{L} \propto \sqrt{L}$ the sample-specific scale at which this last cluster is decimated.

In long finite chains of length $L$, the end-to-end spin correlations $\left\langle\hat{\sigma}_{0}^{z} \hat{\sigma}_{L}^{z}\right\rangle$ are a useful probe of the long length-scaleordering tendencies. The distributions and moments of these can be calculated exactly in the asymptotic limit of long chains and small $\delta$; these compare well with numerical results. ${ }^{6}$ The distributions can be expressed usefully in terms of

$$
\Lambda_{z} \equiv-\log C^{z z}(0, L) .
$$

This logarithm of the correlations scales with $\sqrt{L}$ at the critical point, with a broad distribution on the same scale. The average correlations, however, decay much more slowly: only as $\overline{C^{z z}} \propto 1 / L$.

\section{Ordered and disordered phases}

When $\delta$ is nonzero but small, there are two scaling regimes. At the early stages of the decimation process, clusters and interactions are not "aware" of being noncritical. In this regime, the critical scaling holds. At longer scales, however, there is a crossover to an off-critical regime. The crossover occurs when the typical cluster sizes and bond lengths are of order of the correlation length

$$
\xi \approx \frac{1}{\delta^{2}}
$$

and the log-energy scale is of order

$$
\Gamma_{\times} \sim \frac{1}{\delta}
$$

At scales larger than $\xi$, the behavior is characteristic of one of the two zero-temperature phases, and thus depends on the sign of $\delta$.

At low energies in the ordered and disordered phases, the scaling between energy and length scales is different from that at the critical point. For small $\delta$, in both phases

$$
\Omega \sim \ell^{-z(\delta)}
$$

with the effective dynamical exponent,

$$
z \approx \frac{1}{|\delta|}
$$

near the critical point.

The distributions of the log interactions also change form. In the disordered phase, the distribution of the effective fields does not continue to broaden, and $\beta \sim \Gamma_{\times} \sim 1 / \delta$. But the effective bonds become longer and longer and weaker and weaker with the distribution of the ln J's broadening rapidly, with typical $\zeta \sim \Gamma$. In the disordered phase, the average order-parameter correlations decay exponentially with the correlation length $\xi \approx 1 / \delta^{2}$. Nevertheless, the typical correlations decay much faster; for example, end-to-end correlations of almost all samples decay as $e^{-2 \delta L}$ (Ref. 17). More precisely, as $L \rightarrow \infty$, the distribution of of the scaled logcorrelation function, $\Lambda_{z} / L$, approaches a delta function peaked at $\Lambda_{z} / L=2 \delta$. The average correlations are thus dominated by exponentially rare samples that happen to have anomalously strong exchanges and/or anomalously weak random fields.

In the ordered phase, $\delta<0$, at low-energy scales the ferromagnetic clusters become bigger and bigger, until their size reaches the length of the system. The transverse fields on these clusters concomitantly continue to become more broadly distributed. This tranverse field is the gap between the symmetric and antisymmetric combinations of the two "ordered" states of the cluster. As opposed to the clusters, the remaining bonds stay relatively short at low energies, and their distribution does not continue to broaden. These fields and bonds thus play opposite roles in the two phases; as discussed below, this is a general consequence of duality.

\section{E. Duality}

As for nonrandom classical and quantum Ising models, there is a dual description of the random quantum chain in terms of bond variables. Instead of using the states $|\uparrow\rangle,|\downarrow\rangle$, on each site, one can use the states of the bonds. This is done by assigning $|+\rangle,|-\rangle$ to the bond if the two spins surrounding the bonds are $|\uparrow \uparrow\rangle,|\downarrow \downarrow\rangle$ or $|\downarrow \uparrow\rangle,|\uparrow \downarrow\rangle$ respectively. These are domain-wall variables. In the new Hilbert space, if we choose the quantization axis to be $x$ rather than $z$, the Hamiltonian has the same form, but with $h$ and $J$ exchanged. The duality is summarized in the following table:

$\begin{array}{ccc}\delta & & -\delta \\ J_{n n+1} & & h_{n} \\ h_{n} & J_{n n+1} \\ \hat{\sigma}_{n}^{x} & \hat{\sigma}_{n}^{z} \hat{\sigma}_{n+1}^{z} \\ \hat{\sigma}_{n}^{z} \hat{\sigma}_{n+1}^{z} & \hat{\sigma}_{n}^{z}\end{array}$

If the $\delta$ dependence of the distributions of the random couplings has the form $\rho_{J}(J=X, \delta)=\rho_{h}(h=X,-\delta)$, the random model is self-dual with $\delta \Rightarrow-\delta$. More generally, it will not be 


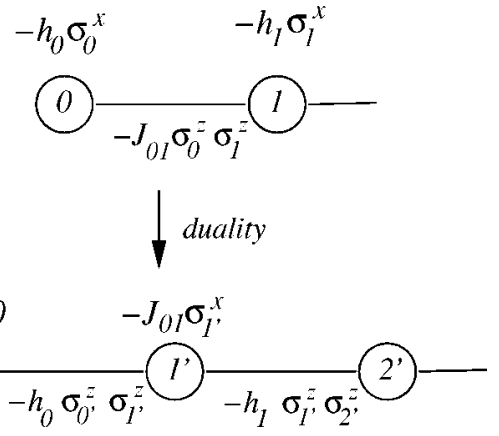

FIG. 2. A chain that terminates with the site 0 is dual to a chain that terminates with a site 0 ', which experiences no transverse field. The bond operator $-h_{0} \hat{\sigma}_{0^{\prime}}^{z} \hat{\sigma}_{1^{\prime}}^{z}$, is then the edge energy operator of the dual chain.

exactly self-dual. But from the definition of $\delta$ in terms of $\rho_{J}$ and $\rho_{h}$ [Eq. (2)], and the universality at low energy scales (in particular, of the distributions of the effective couplings), we expect that the asymptotic behavior at low energies and small $\delta$ will indeed be self-dual for any well-behaved distributions. ${ }^{25}$

As we are interested in end-to-end correlations of finite chains, we must consider what happens to the ends of a chain under the duality transformation. Let $-h_{0} \hat{\sigma}_{0}^{x}$ be the energy operator on the left end-site. Under duality, this site will be mapped into a bond with corresponding energy operator $-h_{0} \hat{\sigma}_{0^{\prime}}^{z} \hat{\sigma}_{1}^{z}$, (see Fig. 2). The bond in the dual chain implies a new site 0 'that corresponds to the domain-wall variable to the left of the first spin in the original chain. This new site, 0 ', thus carries the information about an arbitrarily fixed boundary condition, e.g., $|\uparrow\rangle_{\text {with }}$ respect to which the leftmost domain wall, and hence the original end spin $\hat{\sigma}_{0}^{z}$, is defined; it thus cannot be entirely forgotten. But in the dual Hamiltonian, the operator $\hat{\sigma}_{0^{\prime}}^{x}$, does not appear, so that the dual end transverse field is zero, and $\hat{\sigma}_{0^{\prime}}^{z}$, is time independent. The same is true at the other end, where the extra spin is needed for the original $\hat{\sigma}_{\mathrm{L}}^{x}$ to be defined. Superficially, the dual chain appears to have one extra degree of freedom associated with each end. But the orientation of the dual edge spins is entirely a convention, and therefore, the additional degrees of freedom have no effect.

Note that in the special case in which the original chain starts with a spin to which no transverse field is applied, the duality transformation yields a chain with the first bond turning into a site. More generally, any site in the original chain on which there is no transverse field corresponds under duality to a break in the chain that divides it into two uncoupled parts. In the original variables, there are concomitantly two disconnected subspaces in which the spin that cannot flip has $\sigma_{z}= \pm 1$, respectively. The parts of the chain to the left and to the right of this spin are thus independent of each other.

\section{F. Energy-energy correlations}

Our goal in this paper is to understand the energy density $(E-E)$ correlations of the random chain. These would be particularly interesting at nonequal times, as they would then yield information on the transport of energy which is the only locally conserved quantity in this system. Unfortunately, in the bulk of the chain, correlations are very hard to calculate for reasons discussed in Ref. 3. Therefore, we study the somewhat simpler but closely related quantities: the endto-end correlations of the energy density in finite chains, restricting our analysis to equal time correlations.

Since the Hamiltonian involves two kinds of terms, $J \hat{\sigma}_{0}^{z} \hat{\sigma}_{1}^{z}$ and $h \hat{\sigma}^{x}$, to obtain the $E$ - $E$ correlations we need to calculate three quantities, $\left\langle J_{01} \hat{\sigma}_{0}^{z} \hat{\sigma}_{1}^{z} h_{L} \hat{\sigma}_{L}^{x}\right\rangle, \quad\left\langle h_{0} \hat{\sigma}_{0}^{x} h_{L} \hat{\sigma}_{L}^{x}\right\rangle, \quad$ and $\left\langle J_{01} \hat{\sigma}_{0}^{z} \hat{\sigma}_{1}^{z} J_{L-1} \hat{\sigma}_{L-1}^{z} \hat{\sigma}_{L}^{z}\right\rangle$. However, the duality transformation simplifies matters, since it maps $h_{0} \hat{\sigma}_{0}^{x} h_{L} \hat{\sigma}_{L}^{x}$ at $\delta$ to $J_{01} \hat{\sigma}_{0}^{z} \hat{\sigma}_{1}^{z} J_{L-1} \hat{\sigma}_{L-1}^{z} \hat{\sigma}_{L}^{z}$ at $-\delta$, so we only need to compute one of these quantities. Also, the mixed correlation function is dual to itself, therefore the distribution of $\left\langle J_{01} \hat{\sigma}_{0}^{z} \hat{\sigma}_{1}^{z} h_{L} \hat{\sigma}_{L}^{x}\right\rangle$ must depend on $|\delta|$, and be the same in the two phases. Note that a related single-end quantity, the imaginary time correlation function $\left\langle\hat{\sigma}_{0}^{x}(0) \hat{\sigma}_{0}^{x}(\tau)\right\rangle$, was considered by Iglói et al. ${ }^{18}$

The calculation of the quantities of interest requires an extension of the methods used so far. The primary reason for this is that the energy correlations are dominated by thirdorder perturbative effects at each stage of the decimation, in contrast to the spin correlations, which are controlled by second-order perturbative effects.

To be able to carry out higher-order RG calculations, we develop an approach to the decimation in terms of unitary transformations; this allows one to follow precisely how operators of interest (such as $\hat{\sigma}^{x}$ ) evolve during the RG process. This approach thereby gives a systematic way to deal with higher orders perturbative effects even in problems previously analyzed using second-order perturbation theory. ${ }^{6}$ The unitary-transformations method is developed in the next section (Sec. II).

\section{UNITARY TRANSFORMATION RENORMALIZATION GROUP}

In this section, we develop a perturbative scheme based on unitary transformations that will allow us to separate the various parts of the Hamiltonian and successively simplify the wave functions of the many-body system to a hierarchical product of simple spin-wave functions. Simultaneously, we must keep track of the original operators in order to eventually compute their ground-state correlations.

We begin with the first stage of decimation by constructing the eigenfunction of the highest energy part of the Hamiltonian and transforming it to take into account the lowenergy parts perturbatively. Specifically, the transformation gets rid of the off-diagonal parts that connect states with large energy differences between them. For the Ising chain, this can be done while preserving the form of the Hamiltonian.

Given a Hamiltonian, $\mathcal{H}$, and a many-body ground-state wave function, $|G\rangle$, with

$$
\mathcal{H}|G\rangle=E_{G}|G\rangle .
$$

we can generally make a unitary transformation with a Hermitian operator $S$ and write 


$$
\begin{gathered}
e^{i S} \mathcal{H} e^{-i S} e^{i S}|G\rangle=e^{i S}|G\rangle, \\
\mathcal{H}_{\mathrm{eff}}=e^{i S} \mathcal{H} e^{-i S}, \text { and } e^{i S}|G\rangle=|H\rangle,
\end{gathered}
$$

with the goal to make $|G\rangle$ close to a product of simple wave functions. Such transformations can be used to eliminate-or separate-low-energy parts in the Hamiltonian.

Let $\mathcal{H}=\mathcal{H}_{0}+V$, where $\mathcal{H}_{0}$ is the high-energy part of $\mathcal{H}$ and $V$ is the remaining low-energy parts. The effective Hamiltonian is then

$$
\mathcal{H}_{\text {eff }}=\mathcal{H}_{0}+V+i\left[S, \mathcal{H}_{0}\right]+i[S, V]+\frac{i^{2}}{2 !}\left[S,\left[S, \mathcal{H}_{0}\right]\right]+O\left(S^{3}\right)
$$

If we are able to choose $S$ so that

$$
V+i\left[S, \mathcal{H}_{0}\right]=0,
$$

then $\mathcal{H}_{\text {eff }}$ will contain no first-order terms. The second-order corrections to $\mathcal{H}_{0}$ give rise to effective interactions. We may now solve for the ground state of $\mathcal{H}_{\text {eff }}$, and hence the original $\mathcal{H}$ :

$$
\begin{gathered}
\mathcal{H}_{\text {eff }}|H\rangle=E_{G}|H\rangle \\
|G\rangle=e^{-i S}|H\rangle .
\end{gathered}
$$

Iterating this process separates the higher-energy parts of the Hamiltonian from the lower-energy parts. At each stage, the effective higher-energy parts can be simply diagonalized. The remaining nondiagonalized Hamiltonian only has pieces with energy much lower then the gap of the high-energy section, which was just diagonalized. The ground-state wave function is then constructed perturbatively from the wavefunction $|H\rangle$, which is a hierarchical wave function simply expressible in terms of the ground states of the high-energy parts of the sequence of $\mathcal{H}_{\text {eff }}$ 's. Each term in the hierarchy will be a spin-cluster pointing in the direction of the transverse field. For an example, see Eq. (35) below.

Note that this method is related to the flow equation approach for interacting quantum problems developed by $\mathrm{Ke}$ hrein and Wegner. ${ }^{19,20}$

\section{A. Unitary RG for transverse field ising chain}

We now apply the transformations (15) to successively reduce the maximum energy scale of the random quantum Ising Hamiltonian, thereby obtaining a series of low-energy effective Hamiltonians of the system. We begin by choosing the largest energy coefficient in the Hamiltonian (1) and denote it $\mathcal{H}_{0}$ (with the corresponding coupling the initial energy scale $\Omega_{I}$ ): for example, $\mathcal{H}_{0}=-h_{1} \hat{\sigma}_{1}^{x}$ (see Fig. 3). Let $V$ designate the part of the Hamiltonian that we would like to eliminate. For the above example, we would like to eliminate the parts connecting site 1 to the rest of the chain:

$$
V=-J_{01} \hat{\sigma}_{0}^{z} \hat{\sigma}_{1}^{z}-J_{12} \hat{\sigma}_{1}^{z} \hat{\sigma}_{2}^{z} .
$$

In addition to these two parts, the Hamiltonian also contains the parts involving the remainder of the chain,

$$
\mathcal{H}_{1}=\ldots-J_{-10} \hat{\sigma}_{-1}^{z} \hat{\sigma}_{0}^{z}-h_{0} \hat{\sigma}_{0}^{x}-h_{2} \hat{\sigma}_{2}^{x}-J_{23} \hat{\sigma}_{2}^{z} \hat{\sigma}_{3}^{z}+\ldots
$$
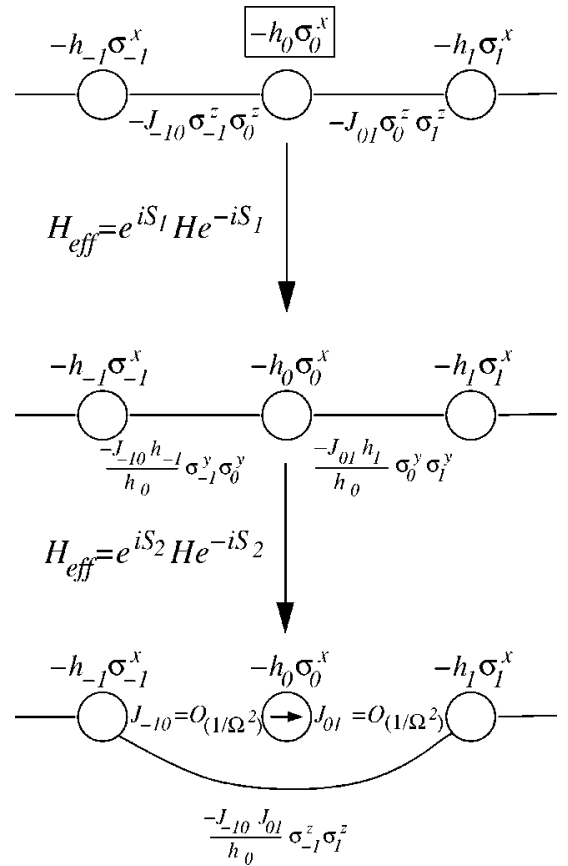

FIG. 3. Site decimation. Spin 0 is almost frozen in the $x$ direction due to the strong magnetic field $h_{0}$. Quantum fluctuations create a second nearest-neighbor effective interaction between sites -1 and 1 . This interaction is weaker than any of $J_{-10}, J_{01}, h_{0}$.

In order to eliminate the first-order couplings to spin $0, S$ must satisfy Eq. (17); thus we first choose

$$
S_{a}=-\frac{J_{01}}{2 h_{1}} \hat{\sigma}_{0}^{z} \hat{\sigma}_{1}^{y}-\frac{J_{12}}{2 h_{1}} \hat{\sigma}_{1}^{y} \hat{\sigma}_{2}^{z},
$$

which yields the following terms in the effective Hamiltonian [Eq. (16)]:

$$
\mathcal{H}_{\mathrm{eff}}=\ldots-\frac{J_{01} J_{12}}{h_{1}} \hat{\sigma}_{0}^{z} \hat{\sigma}_{1}^{x} \hat{\sigma}_{2}^{z}-\frac{h_{0} J_{01}}{h_{1}} \hat{\sigma}_{0}^{y} \hat{\sigma}_{1}^{y}-\frac{h_{2} J_{12}}{h_{1}} \hat{\sigma}_{1}^{y} \hat{\sigma}_{2}^{y}+\ldots
$$

Note that site 1 is still coupled to adjacent sites by a secondorder interaction. We would like to restore the Hamiltonian to its original form; thus we need to eliminate the new type of interaction. To get rid of it, we perform another transformation using

$$
S_{b}=-\frac{h_{0} J_{01}}{2 h_{1}^{2}} \hat{\sigma}_{0}^{y} \hat{\sigma}_{1}^{z}-\frac{h_{2} J_{12}}{2 h_{1}^{2}} \hat{\sigma}_{1}^{z} \hat{\sigma}_{2}^{y} .
$$

The effective Hamiltonian now includes

$$
\begin{gathered}
\mathcal{H}_{\mathrm{eff}}=\ldots-J_{-10} \hat{\sigma}_{-1}^{z} \hat{\sigma}_{0}^{z}-h_{0} \hat{\sigma}_{0}^{x} \\
-\widetilde{J}_{02} \hat{\sigma}_{0}^{z} \hat{\sigma}_{1}^{x} \hat{\sigma}_{2}^{z}-h_{2} \hat{\sigma}_{2}^{x}-J_{23} \hat{\sigma}_{2}^{z} \hat{\sigma}_{3}^{z}+\ldots, \\
\quad-h_{1} \hat{\sigma}_{1}^{x},
\end{gathered}
$$

from which we see that in the low-energy subspace of $\mathcal{H}_{0}$, the effective exchange between spins 0 and 2 is given by 


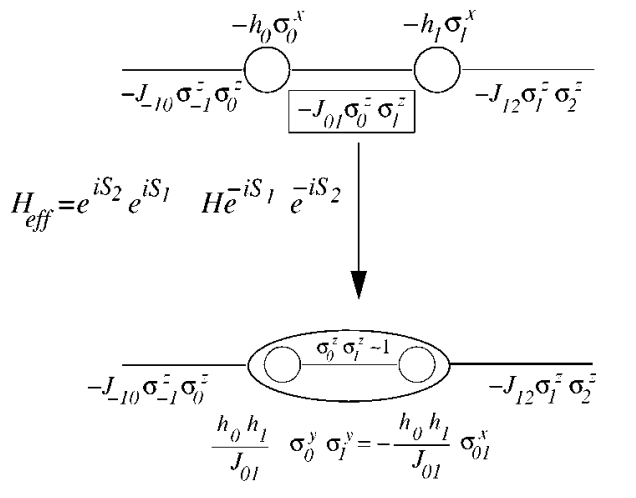

FIG. 4. Bond decimation. Sites 0 and 1 are frozen into one cluster by the strong Ising interaction, $J_{01}$. Quantum fluctuations produce an effective magnetic field, $\tilde{h}_{01}=h_{0} h_{1} / J_{01}$, which flips the composite spin cluster. This field is weaker than any of $h_{0}, h_{1}, J_{01}$.

$$
\tilde{J}_{02}=\frac{J_{01} J_{12}}{h_{1}} .
$$

Since $h_{1}$ is the strongest coupling energy in the chain, the resulting effective bond obeys

$$
\widetilde{J}_{02} \ll h_{0}, J_{01}, J_{12},
$$

where the sharpness of the inequality is because we assume strong randomness. We can now partially diagonalize $\mathcal{H}_{\text {eff }}$ by writing

$$
\begin{gathered}
|H\rangle=|\rightarrow\rangle_{1}\left|\widetilde{G}^{(1)}\right\rangle \\
|G\rangle=e^{-i S_{a}} e^{-i S_{b}}|H\rangle,
\end{gathered}
$$

where $\hat{\sigma}_{1}^{x}\left|\rightarrow_{1}\right\rangle=\left|\rightarrow_{1}\right\rangle$ and $\left|\widetilde{G}^{(1)}\right\rangle$ involves only the spins other than 1 . We are left with a renormalized spin chain with the spin at site 1 eliminated, and with an effective interaction $\bar{J}_{02} \hat{\sigma}_{0}^{z} \hat{\sigma}_{2}^{z}$ between spin 0 and spin 2 . [Note that we could also keep the high-energy sector that involves $\left|\leftarrow_{1}\right\rangle$; the effective Hamiltonian and state of the rest of the chain would differ from those of the low-energy sector because of the presence of $\hat{\sigma}_{(1)}^{x}$ in $\mathcal{H}_{\text {eff }}$ of Eq. (24).]

The analog of the above results for the case where an exchange interaction, e.g., $\mathcal{H}_{0}=-J_{12} \hat{\sigma}_{1}^{z} \hat{\sigma}_{2}^{z}$, is eliminated is (Fig. 4)

$$
\begin{gathered}
\mathcal{H}_{0}=-J_{12} \hat{\sigma}_{1}^{z} \hat{\sigma}_{2}^{z}, \\
V=-h_{1} \hat{\sigma}_{1}^{x}-h_{2} \hat{\sigma}_{2}^{x}, \\
S_{a}=\frac{h_{1}}{2 J_{12}} \hat{\sigma}_{1}^{y} \hat{\sigma}_{2}^{z}+\frac{h_{2}}{2 J_{12}} \hat{\sigma}_{1}^{z} \hat{\sigma}_{2}^{y}, \\
S_{b}=-\frac{h_{0} J_{01}}{2 J_{12}^{2}} \hat{\sigma}_{1}^{y} \hat{\sigma}_{0}^{z}-\frac{h_{3} J_{23}}{2 J_{12}^{2}} \hat{\sigma}_{3}^{z} \hat{\sigma}_{2}^{y} .
\end{gathered}
$$

This could be obtained by using the duality described in the introduction (Sec. I E) and in Ref. 3, or by direct computation. The ground state of $\mathcal{H}_{0}=-J_{12} \hat{\sigma}_{1}^{z} \hat{\sigma}_{2}^{z}$ is doubly degenerate with spins 1 and 2 either in the state $\left|\uparrow_{(12)}\right\rangle=\left|\uparrow_{1}\right\rangle\left|\uparrow_{2}\right\rangle$ or in the state $\left|\downarrow_{(12)}\right\rangle=\left|\downarrow_{1}\right\rangle\left|\downarrow_{2}\right\rangle$. Therefore, in the ground state of $\mathcal{H}_{0}$ $=\hat{\sigma}_{1}^{z} \hat{\sigma}_{2}^{z}$, spins 1 and 2 form a ferromagnetic cluster, which we denote as Eq. (12). We can define cluster operators, $\hat{\sigma}_{(12)}^{z}$ and $\hat{\sigma}_{(12)}^{x}$, that operate on the spin cluster (12) in the following way:

$$
\begin{gathered}
\hat{\sigma}_{1}^{z} \Rightarrow \hat{\sigma}_{(12)}^{z} \\
\hat{\sigma}_{2}^{z} \Rightarrow \hat{\sigma}_{(12)}^{z} \\
-\hat{\sigma}_{1}^{y} \hat{\sigma}_{2}^{y} \Rightarrow \hat{\sigma}_{(12)}^{x},
\end{gathered}
$$

in terms of which

$$
\begin{aligned}
\mathcal{H}_{\mathrm{eff}}-\mathcal{H}_{0}= & \ldots-h_{0} \hat{\sigma}_{0}^{x}-J_{0(12)} \hat{\sigma}_{0}^{z} \hat{\sigma}_{(12)}^{z}-\widetilde{h}_{(12)} \hat{\sigma}_{(12)}^{x} \\
& -J_{(12) 3} \hat{\sigma}_{(12)}^{z} \hat{\sigma}_{3}^{z}-h_{3} \hat{\sigma}_{3}^{x}-\ldots,
\end{aligned}
$$

with

$$
\tilde{h}_{(12)}=\frac{h_{1} h_{2}}{J_{12}}
$$

being the effective transverse field on the new cluster (12) that has replaced the pair of spins 1 and 2 that now only appear separately in the high-energy term in $\mathcal{H}_{0}$. Again, since $J_{12}$ is the strongest energy, and strong randomness is assumed, the effective transverse field obeys:

$$
\tilde{h}_{(12)} \ll h_{1}, h_{2}, J_{12} .
$$

In both the decimation cases we regain the initial form of the Hamiltonian, but with one less spin. As shown in Refs. 2 and 3 , with even stronger randomness. The increase in the randomness with each step justifies the iterative application of the real space $\mathrm{RG}$ as described in this section. In each step, we eliminate a high-energy subspace of the Hilbert space of the chain, which is gapped by $2 \Omega$ from the remaining subspace. The range of excitations in the remaining subspace is much smaller than $\Omega$. The iterative application of the decimation procedure outlined here amounts to separating the Hilbert space of the chain into a hierarchy of sequentially decreasing energy subspaces. If the coupling distributions expand without bounds during the flow, this method is asymptotically exact. ${ }^{26}$

After applying this set of transformation rules $L$ (the original chain length) times, we are left with a single spincluster that carries the moments of some fraction of the set of original spins. The ground state of the chain is then given by the state in which this cluster points in the $x$ direction due to the transverse field. In the same way, we can also access the various excitations of the quantum Ising chain by keeping high-energy subspaces in the decimation process.

At the end of the decimation process, the full effective Hamiltonian is given by 


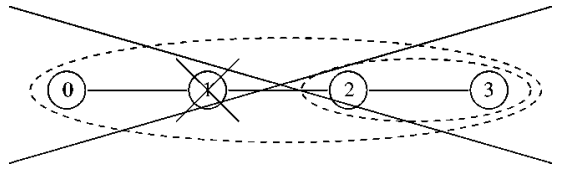

FIG. 5. An example of hierarchical decimation of a chain with four sites. First, site 1 is decimated. At a lower-energy scale, sites 2 and 3 form a ferromagnetic cluster, which we denote (23). Cluster (23) then forms a cluster with site 0 . The last process is a decimation of the cluster (023). The gound-state wave function of this chain is constructed perturbatively from the hierarchical wavefunction $|H\rangle$, which is given in Eq. (35).

$$
\mathcal{H}_{\mathrm{eff}}=e^{i S^{(L)}} e^{i S^{(L-1)}} \ldots e^{i S^{(2)}} e^{i S^{(1)}} \mathcal{H} e^{-i S^{(1)}} e^{-i S^{(2)}} \ldots e^{-i S^{(L-1)}} e^{-i S^{(L)}}
$$

with $S^{(j)}$ representing the transformation of the $j$ th stage of the renormalization. At the final stage the free ground-state wave function, $|H\rangle$, is related to the ground state of the original problem by

$$
|G\rangle=e^{i S^{(1)}} e^{-i S^{(2)}} \ldots e^{-i S^{(L-1)}} e^{-i S^{(L)}}|H\rangle .
$$

Note that the later transformations are the first to operate on $|H\rangle$. In the end of the decimation process, $|H\rangle$ is a product of cluster wave functions, where each cluster points in the direction of the transverse field. For example, $|H\rangle$ for a chain of four spins as in Fig. 5 is given by

$$
|H\rangle=\left|\rightarrow_{(023)}\right\rangle\left|\rightarrow_{1}\right\rangle=\left(\left|\uparrow_{0}\right\rangle\left|\uparrow_{2}\right\rangle\left|\uparrow_{3}\right\rangle+\left|\downarrow_{0}\right\rangle\left|\downarrow_{2}\right\rangle\left|\downarrow_{3}\right\rangle\right)\left(\left|\uparrow_{1}\right\rangle+\left|\downarrow_{1}\right\rangle\right) .
$$

\section{B. Evolution of effective operators}

The quantities we are interested in can be written in terms of $|H\rangle$ and the set of unitary transformations used in the RG process. For example, let us consider

$$
\left\langle\hat{\sigma}_{0}^{x} \hat{\sigma}_{L}^{x}\right\rangle=\left\langle G\left|\hat{\sigma}_{0}^{x} \hat{\sigma}_{L}^{x}\right| G\right\rangle,
$$

with $|G\rangle$ known in terms of $|H\rangle$. We can write

$$
\begin{aligned}
\left\langle G\left|\hat{\sigma}_{0}^{x} \hat{\sigma}_{L}^{x}\right| G\right\rangle & =\left\langle H\left|e^{i S^{(L)}} \ldots e^{i S^{(2)}} e^{i S^{(1)}} \hat{\sigma}_{0}^{x} \hat{\sigma}_{L}^{x} e^{-i S^{(1)}} e^{i S^{(2)}} \ldots e^{-i S^{(L)}}\right| H\right\rangle \\
& =\left\langle H\left|e^{i S^{(L)}} \ldots e^{i S^{(2)}} e^{i S^{(1)}} \hat{\sigma}_{0}^{x} e^{-i S^{(1)}} e^{-i S^{(2)}} \ldots e^{-i S^{(L)}} e^{i S^{(L)}} \ldots e^{i S^{(2)}} e^{i S^{(1)}} \hat{\sigma}_{L}^{x} e^{-i S^{(1)}} e^{i S^{(2)}} \ldots e^{i S^{(L)}}\right| H\right\rangle \\
& =\left\langle H\left|\widetilde{\hat{\sigma}_{0}^{x}} \widetilde{\hat{\sigma}_{L}^{x}}\right| H\right\rangle .
\end{aligned}
$$

Thus, we see that calculating expectation values of an operator $A$ with respect to the ground state $|G\rangle$ is equivalent to calculating the expectation value of the effective operator $\widetilde{A}$ with respect to $|H\rangle$ :

$$
\begin{gathered}
\langle G|A| G\rangle=\langle H|\widetilde{A}| H\rangle \\
\widetilde{A}=e^{i S^{(L)}} \ldots e^{i S^{(2)}} e^{i S^{(1)}} A e^{-i S^{(1)}} e^{-i S^{(2)}} \ldots e^{-i S^{(L)}} .
\end{gathered}
$$

\section{TRANSVERSE FIELD CORRELATIONS}

\section{A. Renormalization of end spin operators}

In order to obtain the transverse field part of the end-toend energy correlations, $\left\langle h_{0} \hat{\sigma}_{0}^{x} h_{L} \hat{\sigma}_{L}^{x}\right\rangle$, we must consider the effects of the two types of renormalization steps (decimation of a site or a bond) on the end operators $\hat{\sigma}_{0}^{x}$ and $\hat{\sigma}_{L}^{x}$. In this section, we show that the decimation of the first bond makes the operator $\hat{\sigma}_{0}^{x}$ evolve to the effective operator $\tilde{\hat{\sigma}}_{0}^{x}$ $=\left(J_{01} / h_{0}\right) \hat{\sigma}_{(01)}^{x}$. We also show that the decimation of the end site yields $\tilde{\hat{\sigma}}_{0}^{x}=\left(J_{01}^{2} h_{1} / h_{0}^{3}\right) \hat{\sigma}_{1}^{x}$, which is third order in the offdiagonal terms that couple high and low energies in the Hamiltonian. In this derivation, we neglect all the subleading contributions to the flow of the edge operators; we show in Appendix A that this is indeed justified.

\section{Decimations away from the ends}

A renormalization step that does not involve the end spin will generally leave the end operators unchanged, since the generator of the unitary transformation of this RG step, $S$, commutes with $\hat{\sigma}_{0}^{x}$. The exception to this is when the decimation involves spins adjacent to the end, for which $\left[S, \hat{\sigma}_{0}^{x}\right] \neq 0$; however, it can be shown that to all orders in perturbation theory, there is no contribution to the dominant parts of the correlation function from the resulting corrections to $\hat{\sigma}_{0}^{x}$.

\section{Decimation of an end bond}

If $J_{01}$ is decimated, sites 0 and 1 will form a cluster (01) (see Fig. 6). The dominant contribution to the correlation function comes from the effective operator $\hat{\sigma}_{(01)}^{x}$. This contribution is obtained from the first order transformation, $S_{a}$ in Eq. (28):

$$
\begin{aligned}
\hat{\sigma}_{0}^{x} & =e^{i S_{a}} \hat{\sigma}_{0}^{x} e^{-i S_{a}}=\hat{\sigma}_{0}^{x}+i\left[\frac{h_{0}}{2 J_{01}} \hat{\sigma}_{0}^{y} \hat{\sigma}_{0}^{z}, \hat{\sigma}_{0}^{x}\right]+i\left[\frac{h_{1}}{2 J_{01}} \hat{\sigma}_{1}^{y} \hat{\sigma}_{0}^{z}, \hat{\sigma}_{0}^{x}\right] \\
& =\hat{\sigma}_{0}^{x}+\frac{h_{0}}{J_{01}} \hat{\sigma}_{0}^{z} \hat{\sigma}_{1}^{z}-\frac{h_{1}}{J_{01}} \overbrace{\hat{\sigma}_{1}^{y}}^{\hat{\sigma}_{(01)}^{y}} .
\end{aligned}
$$

The first two terms in Eq. (39) will not evolve under the continuing renormalization. Their expectation values are 


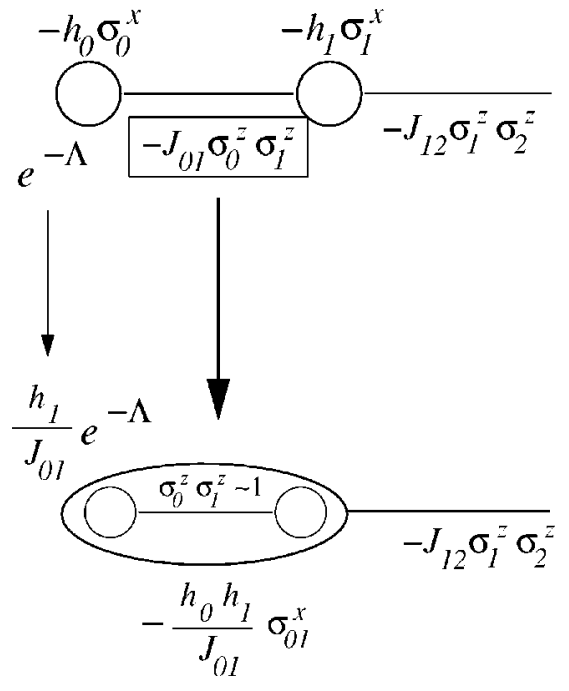

FIG. 6. Evolution of $\hat{\sigma}_{0}^{x}$ in a bond decimation. When the end site forms a cluster with its neighbor, the operator $\sigma_{0}^{x}$ gets renormalized and gains a factor of $h_{1} / J_{01}$.

$$
\begin{gathered}
\left\langle H\left|\hat{\sigma}_{0}^{x}\right| H\right\rangle=0, \\
\left\langle H\left|\hat{\sigma}_{0}^{z} \hat{\sigma}_{1}^{z}\right| H\right\rangle=1 .
\end{gathered}
$$

The only piece of Eq. (39) relevant to us is the third piece. As indicated in Eq. (39), when the operator $-\hat{\sigma}_{1}^{y} \hat{\sigma}_{0}^{y}$ is restricted to the low-energy subspace in which sites 0 and 1 form a cluster, it is equivalent to the cluster operator $\hat{\sigma}_{(01)}^{x}$. Therefore, when the end spin forms a cluster with its neighbor via a bond decimation, the flow of the transverse spin is given by

$$
\hat{\sigma}_{0}^{x} \Rightarrow \frac{h_{1}}{J_{01}} \hat{\sigma}_{(01)}^{x} .
$$

\section{End site decimation}

If $h_{0}$ is the strongest interaction in the chain, site 0 will be decimated. Applying the first-order transformation $S_{a}$ from Eq. (21) to $\hat{\sigma}_{0}^{x}$ yields

$$
\begin{gathered}
S_{a}=-\frac{J_{01}}{2 h_{0}} \hat{\sigma}_{0}^{y} \hat{\sigma}_{1}^{z}, \\
\tilde{\hat{\sigma}_{0}^{x}}=\hat{\sigma}_{0}^{x}+i\left[\frac{j_{01}}{2 h_{0}} \hat{\sigma}_{0}^{y} \hat{\sigma}_{1}^{z}, \hat{\sigma}_{0}^{x}\right]=\hat{\sigma}_{0}^{x}+\frac{J_{01}}{h_{0}} \hat{\sigma}_{0}^{z} \hat{\sigma}_{1}^{z} .
\end{gathered}
$$

Applying the second-order transformation yields

$$
\begin{gathered}
S_{b}=-\frac{h_{1} J_{01}}{2 h_{0}^{2}} \hat{\sigma}_{0}^{z} \hat{\sigma}_{1}^{y} \\
\hat{\sigma}_{0}^{x}=\hat{\sigma}_{0}^{x}-i\left[\frac{h_{1} J_{01}}{2 h_{0}^{2}} \hat{\sigma}_{0}^{z} \hat{\sigma}_{1}^{y}, \hat{\sigma}_{0}^{x}+\frac{J_{01}}{h_{0}} \hat{\sigma}_{0}^{z} \hat{\sigma}_{1}^{z}\right]=\hat{\sigma}_{0}^{x}+\frac{h_{1} J_{01}}{h_{0}^{2}} \hat{\sigma}_{0}^{y} \hat{\sigma}_{1}^{y} \\
+\frac{h_{1} J_{01}^{2}}{h_{0}^{3}} \hat{\sigma}_{2}^{x} .
\end{gathered}
$$

Once again, the first two terms in Eq. (43) do not contribute

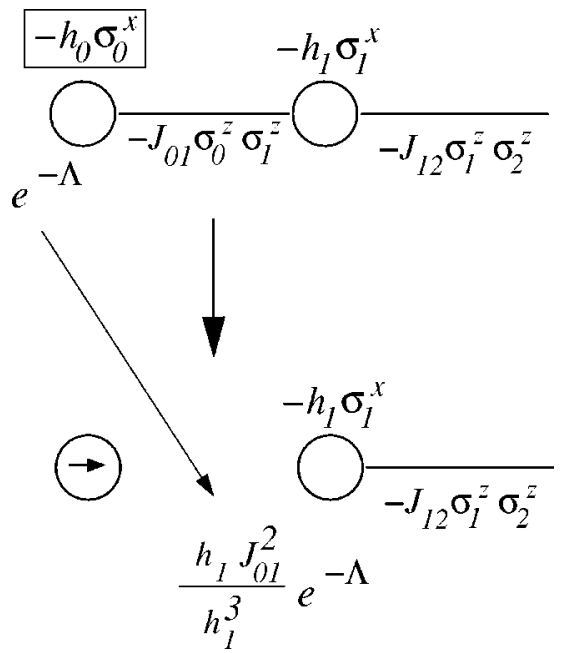

FIG. 7. Evolution of $\sigma_{0}^{x}$ in a site decimation. Although $\sigma_{0}^{x}$ obtains an expectation value, its fluctuations are still influenced by the state of site 1 . This is reflected in the renormalization of $\sigma_{0}^{x}$ as $\left(J_{01}^{2} / h_{0}^{2}\right) \sigma_{1}^{x}$ which gives rise to a factor of $\left(J_{01}^{2} h_{0} / h_{1}^{3}\right)$ in the correlation functions of $\hat{\sigma}_{0}^{x}$.

to truncated correlation functions, since $\left\langle\hat{\sigma}_{0}^{x}\right\rangle=1,\left\langle\hat{\sigma}_{0}^{y}\right\rangle=0$. The flow of $\hat{\sigma}_{0}^{x}$ in this case is

$$
\hat{\sigma}_{0}^{x} \Rightarrow \frac{h_{1} J_{01}^{2}}{h_{0}^{3}} \hat{\sigma}_{1}^{x} .
$$

\section{B. Evolution of the correlation function}

As the renormalization proceeds, the effective operators $\widetilde{\hat{\sigma}_{0}^{x}}$ and $\tilde{\hat{\sigma}_{L}^{x}}$ accrue multiplicative factors that will eventually combine to form the end-to-end correlation function. The evolution of these prefactors is obtained from Eqs. (41) and (44) by the method outlined in Ref. 6.

At log-energy scale $\Gamma$, we write the effective operator of the end spin as

$$
\hat{\sigma}_{0}^{x} \Rightarrow \tilde{\hat{\sigma}}_{0}^{x}(\Gamma) e^{-\Lambda(\Gamma)}
$$

where $\tilde{\hat{\sigma}_{0}^{x}}$ operates on the first spin cluster of the renormalized chain at the scale $\Gamma$. Using the logarithmic variables $\beta$ $=\log (\Omega / h), \zeta=\log (\Omega / J)$, we can rewrite Eqs. (41) and (44) and the results described in Figs. 6 and 7 in terms of $\Lambda$ :

$$
\text { bond-decimation: } \Lambda \Rightarrow \Lambda+\beta_{1} \text {, }
$$

spin-decimation: $\Lambda \Rightarrow \Lambda+\beta_{1}+2 \zeta_{01}$.

The quantities $\Lambda, \beta_{0}$, and $l_{0}^{c}$ (length of the cluster containing the end spin) are correlated at all stages of the renormalization. Therefore, we must keep track of their joint distribution, which we define as

$$
\operatorname{Prob}\left[d l_{0}^{c}, d \beta_{0}, d \Lambda\right]=\omega\left(\beta_{0}, l_{0}^{c}, \Lambda \mid \Gamma\right) d \beta_{0} d l_{0}^{c} d \Lambda .
$$

Using the results of Ref. 3 and Eqs. (28) and (46), we can write the evolution equation for $\omega(\beta, l, \Lambda \mid \Gamma)$ : 


$$
\begin{aligned}
\frac{d \omega(\beta, l, \Lambda \mid \Gamma)}{d \Gamma}= & \frac{\partial \omega(\beta, l, \Lambda \mid \Gamma)}{\partial \beta} \\
& +\int \omega\left(\beta_{0}=0, l_{c}^{0}, \Lambda^{\prime} \mid \Gamma\right) P\left(\zeta_{0}, l_{0}^{b}\right) R\left(\beta_{1}, l_{1}^{c}\right) \delta\left(\Lambda-\Lambda^{\prime}-2 \zeta_{0}-\beta_{1}\right) \delta\left(l-l_{0}^{c}-l_{0}^{b}-l_{1}^{c}\right) \delta\left(\beta-\beta_{1}\right) d l_{0}^{c} d l_{0}^{b} d l_{1}^{c} d \Lambda^{\prime} d \beta_{1} d \zeta_{0} \\
& +\int \omega\left(\beta_{0}, l_{0}^{c}, \Lambda^{\prime} \mid \Gamma\right) R\left(\beta_{1}, l_{1}^{c}\right) P\left(\zeta_{0}=0, l_{0}^{b}\right) \delta\left(\Lambda-\Lambda^{\prime}-\beta_{1}\right) \delta\left(l-l_{0}^{c}-l_{0}^{b}-l_{1}^{c}\right) \delta\left(\beta-\beta_{1}-\beta_{2}\right) d l_{0}^{c} d l_{0}^{b} d l_{1}^{c} d \Lambda^{\prime} d \beta_{0} d \beta_{1} \\
& -\int P\left(0, l^{\prime}\right) d l^{\prime} \omega(\beta, l, \Lambda \mid \Gamma)
\end{aligned}
$$

in terms of the distributions $P$ and $R$ of the log couplings and lengths of the bonds and spin-clusters at scale $\Gamma$. Employing the notation for convolutions introduced in Ref. $3, f\left(x_{1}\right) \stackrel{x}{\otimes} g\left(x_{2}\right)=\int f\left(x_{1}\right) g\left(x_{2}\right) \delta\left(x-x_{1}-x_{2}\right) d x_{1} d x_{2}$, we can write the above equation in a more compact way:

$$
\begin{aligned}
\frac{d \omega(\beta, l, \Lambda \mid \Gamma)}{d \Gamma}= & \frac{\partial \omega(\beta, l, \Lambda \mid \Gamma)}{\partial \beta}+\int \omega\left(\beta_{0}=0, l_{c}^{0}, \Lambda^{\prime} \mid \Gamma\right) \otimes P\left(\zeta_{0}, l_{0}^{b}\right) \otimes R\left(\beta, l_{1}^{c}\right) \delta\left(\Lambda-\Lambda^{\prime}-2 \zeta_{0}-\beta\right) d \Lambda^{\prime} d \zeta_{0} \\
& +\int \omega\left(\beta_{0}, l_{0}^{c}, \Lambda^{\prime} \mid \Gamma\right) \otimes R\left(\beta_{1}, l_{1}^{c}\right)^{l} \otimes P\left(\zeta_{0}, l_{0}^{b}\right) \delta\left(\Lambda-\Lambda^{\prime}-\beta_{1}\right) d \Lambda^{\prime}-\int P\left(0, l^{\prime}\right) d l^{\prime} \omega(\beta, l, \Lambda \mid \Gamma) .
\end{aligned}
$$

By Laplace transforming $\omega(\beta, l, \Lambda)$, with respect to both $l$ and $\Lambda$,

$$
\omega(\beta, y, \lambda)=\int d l \int d \Lambda e^{-l y} e^{-\lambda \Lambda} \omega(\beta, l, \Lambda),
$$

we obtain

$$
\begin{aligned}
\frac{d \omega(\beta, y, \lambda \mid \Gamma)}{d \Gamma}= & \frac{\partial \omega(\beta, y, \lambda)}{\partial \beta} \\
& +\omega\left(\beta_{0}=0, y, \lambda \mid \Gamma\right) \int e^{-2 \zeta \lambda} P(\zeta, y) d \zeta R(\beta, y) \\
& +\int \omega\left(\beta_{0}, y, \lambda\right) \otimes R\left(\beta_{1}, y\right) P\left(\zeta_{0}=0, y\right) \\
& -P(0, y=0) \omega(\beta, y, \lambda) .
\end{aligned}
$$

In Ref. 3, the scaling limits of the functions $R(\beta, y), P(\beta, y)$ are derived:

$$
\begin{gathered}
P(\zeta, y \mid \Gamma)=\Upsilon(y, \Gamma) e^{-\zeta u(y, \Gamma),} \\
R(\beta, y \mid \Gamma)=T(y, \Gamma) e^{-\beta \tau(y, \Gamma)}, \\
\Upsilon(y, \Gamma)=\frac{\Delta(y)}{[\sinh \Delta(y) \Gamma]} e^{-\delta \Gamma,} \\
\tau(y, \Gamma)=\delta+\Delta(y) \operatorname{coth}[\Delta(y) \Gamma], \\
u(y, \Gamma)=-\delta+\Delta(y) \operatorname{coth}[\Delta(y) \Gamma], \\
\Delta(y)=\sqrt{y+\delta^{2}} .
\end{gathered}
$$

Using these results and the corresponding notations, we get

$$
\begin{aligned}
\frac{d \omega(\beta, y, \lambda)}{d \Gamma}= & \frac{\partial \omega(\beta, y, \lambda)}{\partial \beta}+\omega(0, \lambda, y) \frac{T(y, \Gamma) \Upsilon(y, \Gamma)}{2 \zeta+u} \\
& +\Upsilon(y, \Gamma) \int \omega\left(\beta_{0}, y, \lambda\right) e^{-\tau \beta_{1}-\lambda \beta_{1}} T(y, \Gamma) \\
& \times \delta\left(\beta-\beta_{0}-\beta_{1}\right) d \beta_{0} d \beta_{1}-\Upsilon(0, \Gamma) \omega(\beta, y, \lambda)
\end{aligned}
$$

To solve this we write $\omega(\beta, y, \lambda)$ in the following form:

$$
\omega(\beta, y, \lambda)=W(y, \lambda) e^{-\beta \lambda-\beta \tau},
$$

under which Eq. (53) becomes

$$
\frac{d W}{d \Gamma}=-(\tau+\lambda) W+\frac{\Upsilon(y, \Gamma) T(y, \Gamma)}{2 \lambda+u} W-\Upsilon(0, \Gamma) W .
$$

Using the definitions of the functions $\Upsilon, T, u$, and $\tau$, we can integrate Eq. (55) and find

$$
W=W_{0} e^{(\lambda+\delta)\left(\Gamma-\Gamma_{I}\right)} \frac{\sinh \left[\Delta(y) \Gamma_{I}\right]}{\sinh [\Delta(y) \Gamma]}\left(\frac{2 \lambda+u\left(y, \Gamma_{I}\right)}{2 \lambda+u(y, \Gamma)}\right) \frac{\tau(0, \Gamma)}{\tau\left(0, \Gamma_{I}\right)} .
$$

The value of $W_{0}$ can be found from the normalization condition on the distribution $\omega(\beta, l, \Lambda)$. To be a properly normalized, $\omega(\beta, l, \Lambda)$ has to obey

$$
\int_{0}^{\infty} d \beta \omega(\beta, y=0, \lambda=0)=1 .
$$


Before proceeding, we should check what initial conditions this distribution satisfies. Setting $\Gamma \Rightarrow \Gamma_{I}$ and $y \Rightarrow 0$, we see

$$
\omega\left(\beta, 0, \lambda \mid \Gamma_{I}\right)=\tau\left(0, \Gamma_{I}\right) e^{-\tau\left(y, \Gamma_{I}\right) \beta} e^{-\lambda \beta}
$$

The inverse Laplace transform in $\lambda$ yields

$$
\omega\left(\beta, 0, \Lambda \mid \Gamma_{I}\right)=\tau\left(0, \Gamma_{I}\right) e^{-\tau\left(0, \Gamma_{I}\right) \beta} \delta(\Lambda-\beta) .
$$

The delta function in Eq. (59) shows that the initial value of the correlation variable is the same as the transverse field, as it should be for this part of the energy-energy correlations.

\section{Last decimation step}

After carrying out the decimation process $L-2$ times and following the flow of the edge energy operators, we end up with two clusters. The remaining clusters correspond to the left and right edges of the chain, and each has a distribution $\omega(\beta, l, \Lambda \mid \Gamma)$ associated with it. In the next decimation step, one of these clusters is decimated, and a single cluster forms. At this stage, we can compute the distribution of the energy correlations from the flow of edge operators and the remaining couplings. Note that the $\Gamma$ at which a single cluster forms is the logarithm of the gap between the first and second excited states (as was also noted in Ref. 18).

The computation of the truncated correlation function is as follows:

$$
\begin{aligned}
C_{L}^{x x} & =\left\langle G\left|h_{0} \hat{\sigma}_{0}^{x} h_{L} \hat{\sigma}_{L}^{x}\right| G\right\rangle-\left\langle G\left|h_{0} \hat{\sigma}_{0}^{x}\right| G\right\rangle\left\langle G\left|h_{L} \hat{\sigma}_{L}^{x}\right| G\right\rangle \\
& =\left\langle H\left|e^{-\Lambda_{\ell}} \hat{\sigma}_{\tilde{\ell}}^{x} \hat{\sigma}_{\widetilde{r}}^{x} e^{-\Lambda_{r}}\right| H\right\rangle-\left\langle H\left|e^{-\Lambda_{\ell}} \hat{\sigma}_{\tilde{\ell}}^{x}\right| H\right\rangle\left\langle H\left|\hat{\sigma}_{\widetilde{r}}^{x} e^{-\Lambda_{r}}\right| H\right\rangle,
\end{aligned}
$$

where $\Lambda_{\ell}$ and $\Lambda_{r}$ are the correlation factors picked up in the RG process, Eq. (46), for the left end and right end transverse spins, respectively, and we have labeled the last remaining spin clusters $\ell$ and $r$. The correlation function can also be written as a sum over excited states:

$$
\begin{aligned}
C_{L}^{x x} & =\left\langle h_{0} \hat{\sigma}_{0}^{x} h_{L} \hat{\sigma}_{L}^{x}\right\rangle-\left\langle h_{0} \hat{\sigma}_{0}^{x}\right\rangle\left\langle h_{L} \hat{\sigma}_{L}^{x}\right\rangle \\
& =\left\langle G\left|\left(h_{0} \hat{\sigma}_{0}^{x}-\left\langle h_{0} \hat{\sigma}_{0}^{x}\right\rangle\right)\left(h_{L} \hat{\sigma}_{L}^{x}-\left\langle h_{L} \hat{\sigma}_{L}^{x}\right\rangle\right)\right| G\right\rangle \\
& =\sum_{\psi \neq G}\left\langle G\left|h_{0} \hat{\sigma}_{0}^{x}\right| \psi\right\rangle\left\langle\psi\left|h_{L} \hat{\sigma}_{L}^{x}\right| G\right\rangle \\
& =e^{-\Lambda_{\ell}-\Lambda_{r}} \sum_{\psi \neq G}\left\langle H\left|\hat{\sigma}_{0}^{x}\right| \psi\right\rangle\left\langle\psi\left|\hat{\sigma}_{1}^{x}\right| H\right\rangle,
\end{aligned}
$$

where the sum over $\psi$ runs over all states except the ground state.

At the penultimate decimation step two processes are possible:

\section{Bond Decimation}

The two remaining clusters become a single cluster, and the only remaining coupling is the transverse field, which makes the combined cluster point in the $x$ direction:

$$
\left|H^{(L)}\right\rangle=\frac{1}{\sqrt{2}}\left(|\uparrow \tilde{\ell}\rangle\left|\uparrow_{\tilde{r}}\right\rangle+|\downarrow \tilde{\ell}\rangle\left|\downarrow_{\tilde{r}}\right\rangle\right),
$$

where $\tilde{\ell}$ and $\tilde{r}$ represent the end clusters at this final stage of the RG. This yields Eq. (60)

$$
C_{L}^{x x}=e^{-\Lambda_{\ell}-\Lambda_{r}}=e^{-\Lambda},
$$

hence

$$
\Lambda=\Lambda_{r}+\Lambda_{\ell},
$$

where $\Lambda$ is the desired log of the energy correlations.

\section{Site Decimation}

In the case of a site decimation it is unimportant which of the last surviving clusters gets decimated. Let us assume that it is the left cluster, $\tilde{\ell}$. This involves the unitary transformation (dropping the tildes) $S_{a}=-\left(J_{\ell r} / h_{\ell}\right) \hat{\sigma}_{\widetilde{\ell}}^{y} \hat{\sigma}_{\widetilde{r}}^{z}$, and makes the ground state $|H\rangle$ be

$$
\mathcal{H}^{(L)}=\left|\rightarrow_{\ell}\right\rangle\left|\rightarrow_{r}\right\rangle=\frac{1}{2}\left(\left|\uparrow_{\ell}\right\rangle+\left|\downarrow_{\ell}\right\rangle\right)\left(\left|\uparrow_{r}\right\rangle+\left|\downarrow_{r}\right\rangle\right) .
$$

By using the sum form in Eq. (61) and applying $S_{a}$ we get

$$
\begin{aligned}
C_{L}^{x x} e^{\Lambda_{l}+\Lambda_{r}} & \approx \sum_{\psi \neq G}\left\langle H\left|e^{i S_{a}} \hat{\sigma}_{\ell}^{x} e^{-i S_{a} \mid}\right| \psi\right\rangle\langle\psi| e^{i S_{a}} \hat{\sigma}_{r}^{x} e^{-i S_{a}|H\rangle} \\
& \approx \sum_{\psi \neq G}\left\langle H\left|-\frac{J_{\ell r}}{h_{\ell}} \hat{\sigma}_{\ell}^{z} \hat{\sigma}_{r}^{z}\right| \psi\right\rangle\left\langle\psi\left|\frac{J_{\ell r}}{h_{\ell}} \hat{\sigma}_{\ell}^{y} \hat{\sigma}_{r}^{y}\right| H\right\rangle=\frac{J_{\ell r}^{2}}{h_{\ell}^{2}} .
\end{aligned}
$$

This yields

$$
\Lambda=\Lambda_{r}+\Lambda_{\ell}+2 \zeta_{\ell} \tilde{r}
$$

The analog of Eq. (25) in Ref. 6 for the measures defined here is

$$
\begin{aligned}
d \operatorname{Prob} & \left(\Lambda_{\ell}, l_{\ell}, \beta_{\ell}, l_{1}^{b}, \zeta_{1}, l_{1}^{c}, \beta_{1}, l_{2}^{b}, \zeta_{2}, \ldots, l_{r}, \beta_{r}, \Lambda_{r} \mid L, \Gamma\right) \\
= & a_{\Gamma} \omega\left(\beta_{\ell}, \Lambda_{\ell}, l_{\ell}\right) P\left(\zeta_{1}, l_{1}^{b}\right) R\left(\beta_{1}, l_{1}^{c}\right) P\left(\zeta_{2}, l_{2}^{b}\right) \ldots \\
& \omega\left(\beta_{r}, \Lambda_{r}, l_{r}\right) \delta\left(l_{\ell}+l_{1}^{b}+l_{1}^{c}+l_{1}^{b}+\ldots+l_{r}-L\right) \\
& \times d\left\{\beta_{i}\right\} d\left\{\zeta_{i}\right\} d \Lambda_{r} d \Lambda_{\ell} d\left\{l_{i}\right\},
\end{aligned}
$$

with

$$
\frac{1}{a_{\Gamma}} \frac{d a_{\Gamma}}{d \Gamma}=\int_{0}^{\infty}[P(0, l)+R(0, l)] d l .
$$

Let us now define the function $J(\Lambda, \Gamma \mid L)$ as

$d$ Prob[chain of length $L$ becomes a single cluster at

$$
\left.\Gamma \text { with } \log \left(C_{L}\right)=\Lambda\right]=J(\Lambda, \Gamma \mid L) d \Gamma d \Lambda .
$$

The probability distribution of $\log \left(C_{L}\right)$ is then given by

$$
f(\Lambda, L)=\int_{0}^{\infty} J(\Lambda, \Gamma \mid L) d \Gamma .
$$


The function $J(\Lambda, \Gamma \mid L)$ has two contributions, the first contribution cames from the case of the penultimate decimation being a bond decimation [Eq. (64)]. The second contribution comes from the case of a site decimation [Eq. (67)]. The combination of the two contribution yields

$$
\begin{aligned}
J(\Lambda, \Gamma \mid L)= & a_{\Gamma} \int P\left(0, l_{\ell r}^{b}\right) \omega\left(\beta_{\ell}, \Lambda_{\ell}, l_{\ell}\right) \omega\left(\beta_{r}, \Lambda_{r}, l_{r}\right) \delta\left(l_{\ell}+l_{\ell r}^{b}\right. \\
& \left.+l_{r}-L\right) \delta\left(\Lambda-\Lambda_{\ell}-\Lambda_{r}\right) d \Lambda_{\ell} d \Lambda_{r} d l_{\ell} d l_{\ell r}^{b} d l_{r} d \beta_{r} d \beta_{\ell} \\
& +2 a_{\Gamma} \int P\left(\zeta_{r}, l_{\ell r}^{b}\right) \omega\left(0, \Lambda_{\ell}, l_{\ell}\right) \omega\left(\beta_{r}, \Lambda_{r}, l_{r}\right) \\
& \times \delta\left(l_{\ell}+l_{\ell r}^{b}+l_{r}-L\right) \delta\left(\Lambda-\Lambda_{\ell}-\Lambda_{r}-2 \zeta_{r}\right) \\
& \times d \Lambda_{\ell} d \Lambda_{r} d l_{\ell} d l_{\ell r}^{b} d l_{r} d \beta_{r} d \zeta_{r},
\end{aligned}
$$

where $l_{\ell, r}^{b}$ is the length of the effective bond connecting the last two clusters.

The Laplace transform of $J$ is considerably simpler to write

$$
\begin{aligned}
J(\Lambda, \Gamma, y)= & a_{\Gamma}\left[P(0, y)\left(\int \omega(\beta, \lambda, y) d \beta\right)^{2}\right. \\
& \left.+2 \int e^{-2 \zeta \lambda} P(\zeta, y) d \zeta \omega\left(0, \Lambda_{\ell}, l_{\ell}\right) \int \omega(\beta, \lambda, y) d \beta\right] \\
= & a_{\Gamma} P(0, y) \frac{\omega(\lambda, \Gamma)^{2}}{(\tau(y \mid \Gamma)+\lambda)}\left[\frac{1}{(\tau(y \mid \Gamma)+\lambda)}\right. \\
& \left.+2 \frac{1}{(u(y \mid \Gamma)+2 \lambda)}\right] .
\end{aligned}
$$

Equation (73) is one of the main results of this paper; from it we will derive the typical and average correlation functions, as well as information on the distribution of $C_{L}$.

\section{EXCHANGE ENERGY AND CROSS CORRELATIONS}

\section{A. Boundaries and duality}

The calculation of the $h \hat{\sigma}_{0}^{x}-h \hat{\sigma}_{L}^{x}$ correlations in the previous section was simplified greatly because the only operators $\hat{\sigma}_{0, L}^{x}$ considered were end operators. In this section, we calculate expressions for the end-to-end correlations of the exchange energy density, $J \hat{\sigma}^{z} \hat{\sigma}^{z}$. We consider the special case for which one or both end transverse fields, $h_{0}$ and $h_{L}$, are zero, which simplifies the calculation considerably. We will argue that the universal features of the correlations will be the same as in the general case with nonzero end fields.

The simplifications with vanishing end transverse-fields arise because this makes the exchange energy be an edge operator in the sense that it is the first and last energy operator in the Hamiltonian:

$$
\mathcal{H}=-J_{01} \hat{\sigma}_{0}^{z} \hat{\sigma}_{1}^{z}-h_{1} \hat{\sigma}_{1}^{x}-\ldots-h_{L-1} \hat{\sigma}_{L-1}^{x}-J_{L-1} \hat{\sigma}_{L-1}^{z} \hat{\sigma}_{L}^{z} .
$$

An edge bond is the dual of an edge site, and, therefore, the edge-to-edge exchange-energy correlations of the ground state of the Hamiltonian in Eq. (74) are duals to the transverse-spin correlations calculated in Sec. III. This is explained below.

As was explained in the introduction (Sec. IE), the duality transforms a bond to a spin, and vice versa. In the previous section we considered a chain that terminates with a site that has a finite transverse field on it, $h_{0}>0$. The dual of this edge is a chain edge that terminates with a nonzero bond, $J_{0^{\prime} 1^{\prime}}=h_{0}$. The site $1^{\prime}$ is the dual of the bond $J_{01}$ and, therefore experiences a field $h_{1^{\prime}}=J_{01}$. Since there is no bond $J_{-10}$, i.e., $J_{-10}=0$, the field on site $0^{\prime}$ is zero as well (see Fig. 2).

In what follows, we calculate the end-to-end correlations of the exchange energy and the cross correlations between the transverse spin and the exchange energy. In both cases we will assume that the chain terminates with the energy operators whose correlations we calculate [as in Eq. (74) for the exchange-energy correlations]. In the case of exchangeenergy correlations, both edges of the chain we consider will terminate with a vanishing transverse field, $h_{0}=h_{L}=0$. Similarly, when we calculate the edge correlations between the exchange energy of sites 0 and 1 , and the transverse spin on site $L$, the edge transverse field $h_{0}$ is set to 0 . These rules allow us to use the dual of the function $\omega(\beta, l, \Lambda \mid \Gamma)$ which was derived in Sec. III B. We define the function $\phi(\zeta, l, \Lambda \mid \Gamma)$ as the dual of $\omega(\beta, l, \Lambda \mid \Gamma)$. This function will keep track of the correlations and evolution of the operator $J_{01} \hat{\sigma}_{0}^{z} \hat{\sigma}_{1}^{z}$ in the same way that $\omega(\beta, l, \Lambda \mid \Gamma)$ was used to keep track of the correlations and evolution of the operator $h_{0} \hat{\sigma}_{0}^{x}$.

The calculations carried out in this section assume that one or both edge transverse fields are zero, but this does not limit the generality of our results for universal quantities. We expect that when the edge transverse fields are nonzero, the correlations of the last bond in a chain will only be modified by a nonuniversal multiplicative factor from the correlations in the special case with no transverse field on the end spin.

\section{B. Evolution of edge exchange-Energy Operator}

As discussed above, the edge exchange energy $J_{01} \hat{\sigma}_{0}^{z} \hat{\sigma}_{1}^{z}$ is dual to the edge transverse field operator $h_{0} \hat{\sigma}_{0}^{x}$. Therefore, we can obtain the distribution function for the evolution of the edge exchange-energy operator from the results of Sec. III.

By making use of the duality (Sec. I E), we can transform all the results obtained in Sec. III to the dual chain. As stated above, we define the analog of $\omega(\beta, l, \Lambda \mid \Gamma)$ to be $\phi(\zeta, l, \Lambda \mid \Gamma): \phi(\zeta, l, \Lambda \mid \Gamma)$ keeps track of the bond strength of the end bond, its length (including the length of the $h=0$ end site), and the $\log$ contribution to the correlation, $\Lambda$. $\phi(\zeta, l, \Lambda \mid \Gamma)$ is obtained from the dual of Eq. (56):

$$
\begin{gathered}
\phi(\zeta, y, \lambda)=\Phi(y, \lambda) e^{-\zeta \lambda-\zeta u(y, \Gamma)}, \\
\phi(y, \lambda)=e^{(\lambda-\delta)\left(\Gamma-\Gamma_{I}\right)} \frac{\sinh \left[\Delta(y) \Gamma_{I}\right]}{\sinh [\Delta(y) \Gamma]} \frac{2 \lambda+\tau\left(y, \Gamma_{I}\right)}{2 \lambda+\tau(y, \Gamma)} u(0, \Gamma) .
\end{gathered}
$$

\section{Exchange-energy correlations}

The results for the exchange-energy correlations are given, by duality, by Eq. (73), with $\delta \rightarrow-\delta$. This yields 


$$
\begin{aligned}
J(\Lambda, \Gamma, y)= & a_{\Gamma} R(0, y) \frac{\Phi(y, \lambda)^{2}}{(u(y \mid \Gamma)+\lambda)}\left(\frac{1}{(u(y \mid \Gamma)+\lambda)}\right. \\
& \left.+2 \frac{1}{(\tau(y \mid \Gamma)+2 \lambda)}\right) .
\end{aligned}
$$

Since the results for the exchange-energy correlations are identical to that of the transverse correlations, we will only analyze the later.

\section{Cross correlations-last decimation step and final expression}

In order to obtain the cross correlations, we need to combine the results for the edge transverse spin flow and exchange-energy flow. In analogy to Sec. III, putting together the two flows happens in the penultinate step of the RG flow. The accumulated multiplicative factors, along with the couplings of the renormalized chain just before it is completely decimated, will determine the total correlations between the transverse spin and exchange energy.

In contrast to Sec. III, the last needed step of the RG to obtain the cross correlations involves the transverse spin of one of the two clusters, and the bond between them:

$$
\begin{aligned}
C_{L}^{x-B}= & \left\langle G\left|h_{0} \hat{\sigma}_{0}^{x} J_{L-1 L} \hat{\sigma}_{L-1}^{z} \hat{\sigma}_{L}^{z}\right| G\right\rangle-\left\langle G\left|h_{0} \hat{\sigma}_{0}^{x}\right| G\right\rangle \\
& \times\left\langle G\left|J_{L-1 L} \hat{\sigma}_{L-1}^{z} \hat{\sigma}_{L}^{z}\right| G\right\rangle \\
= & \left\langle H\left|e^{-\Lambda_{\ell}} \hat{\sigma}_{\tilde{\ell}}^{x} \hat{\sigma}_{\tilde{\ell}}^{z} \hat{\sigma}_{\widetilde{r}}^{z} e^{-\Lambda_{r}}\right| H\right\rangle-\left\langle H\left|e^{-\Lambda_{\ell}} \hat{\sigma}_{\tilde{\ell}}^{x}\right| H\right\rangle \\
& \times\left\langle H\left|\hat{\sigma}_{\tilde{\ell}}^{z} \hat{\sigma}_{\widetilde{r}}^{z} e^{-\Lambda_{r}}\right| H\right\rangle,
\end{aligned}
$$

where $B$ stands for bond. The two possibilities for the last step of the decimation process are the decimation of the bond $\left(J_{\tilde{\ell} \tilde{r}}\right)$, or of the $\ell$ cluster $\left(h_{\tilde{\ell}}\right)$. These two processes are dual to each other; hence we only need to consider one of them. Let us consider the site decimation.

As before, the ground state will be

$$
|H\rangle=|\rightarrow \tilde{\ell}\rangle\left|\rightarrow_{\tilde{r}}\right\rangle=\frac{1}{2}\left(\left|\uparrow_{\tilde{\ell}}\right\rangle+\left|\downarrow_{\ell}\right\rangle\right)\left(\left|\uparrow_{\tilde{r}}\right\rangle+\left|\downarrow_{\tilde{r}}\right\rangle\right) .
$$

From the transformation $S_{a}=-\left(J_{\ell r} / h_{\ell}\right) \hat{\sigma}_{\tilde{r}}^{z} \hat{\sigma}_{\tilde{\ell}}^{y}$ that induces this decimation, the correlations are found to be:

$$
\begin{aligned}
& C_{L}^{x-B} e^{\Lambda_{\ell}+\Lambda_{r}}=\left\langle H\left|e^{i S_{a}} \hat{\sigma}_{\tilde{\ell}}^{x} e^{-i S_{a}} e^{i S_{a}} \hat{\sigma}_{\tilde{\ell}}^{z} \hat{\sigma}_{\tilde{r}}^{z} e^{-i S_{a}}\right| H\right\rangle \\
& -\left\langle H\left|e^{i S_{a}} \hat{\boldsymbol{\sigma}}_{\tilde{\ell}}^{x} e^{-i S_{a}}\right| H\right\rangle\left\langle H\left|e^{i S_{a}} \hat{\boldsymbol{\sigma}}_{\tilde{\ell}}^{z} \hat{\sigma}_{\tilde{r}}^{z} e^{-i S_{a}}\right| H\right\rangle \\
& =\left\langle H\left|\left(\hat{\sigma}_{\tilde{\ell}}^{x}-\frac{J_{\ell r}}{\widetilde{h}_{\ell}} \hat{\sigma}_{\tilde{\ell}}^{z} \hat{\sigma}_{\tilde{r}}^{z}\right)\left(\hat{\sigma}_{\tilde{\ell}}^{z} \hat{\sigma}_{\tilde{r}}^{z}+\frac{J_{\ell r}}{\widetilde{h}_{\ell}} \hat{\sigma}_{\tilde{\ell}}^{x}\right)\right| H\right\rangle \\
& -\left\langle H\left|\left(\hat{\sigma}_{\tilde{\ell}}^{x}-\frac{J_{\ell \underline{\ell}}}{\widetilde{h}_{\ell}} \hat{\sigma}_{\tilde{\ell}}^{z} \hat{\sigma}_{\tilde{r}}^{z}\right)\right| H\right\rangle\left\langle H\left|\left(\hat{\sigma}_{\tilde{\ell}}^{z} \hat{\sigma}_{\widetilde{r}}^{z}+\frac{J_{\ell r}}{\widetilde{h}_{\ell}} \hat{\sigma}_{\tilde{\ell}}^{x}\right)\right| H\right\rangle \\
& =-\frac{J_{\tilde{\ell} \tilde{r}}}{\tilde{h}_{\ell}}=-e^{-\tilde{\zeta}_{\ell} \tilde{r}} .
\end{aligned}
$$

Thus, the cluster decimation process yields

$$
\Lambda=\Lambda_{\ell}+\Lambda_{r}+\zeta_{\tilde{\ell} \tilde{r}}
$$

By duality, the bond decimation process yields

$$
\Lambda=\Lambda_{\ell}+\Lambda_{r}+\beta_{\ell} \text {. }
$$

Following the reasoning that led to Eqs. (72) and (73) we get:

$$
J(\lambda, \Gamma \mid y)=a_{\Gamma} \omega(\lambda, \Gamma) \phi(\lambda, \Gamma)\left(\frac{1}{\tau(y \mid \Gamma)+\lambda}+\frac{1}{u(y \mid \Gamma)+\lambda}\right) .
$$

This is the second main result in this paper, and it is analogous to Eq. (73). Here we must bear in mind that the correlations obtained here are negative [see Eq. (79)]. This is to be expected, since the two operators, $\hat{\sigma}^{x}$ and $\hat{\sigma}^{z} \hat{\sigma}^{z}$, try to impose competing orders; one tends to make each spin point in the $x$ direction, whereas the other tends to create a cluster of spins that point in the $z$ direction.

\section{RESULTS}

The above results (73) and (82) in principal allow the calculation of the distribution function for the log correlations, $\Lambda=-\log \left(C_{L}\right)$, of long finite chains. In the following sections we calculate the average correlations, $\overline{C_{L}}$, and the distribution, $f(\Lambda \mid L)$, for all $\delta$.

\section{A. Average $\boldsymbol{h} \hat{\boldsymbol{\sigma}}^{x}-\boldsymbol{h} \hat{\boldsymbol{\sigma}}^{x}$ and $\boldsymbol{J} \hat{\boldsymbol{\sigma}}^{z} \hat{\boldsymbol{\sigma}}^{z}-J \hat{\boldsymbol{\sigma}}^{z} \hat{\boldsymbol{\sigma}}^{z}$ correlations}

\section{Derivation of the Average}

In this section we derive the average $x$ - $x$ correlations. The $B B$ correlations of the exchange energy are obtained from the $x-x$ correlations upon the transformation $\delta \rightarrow-\delta$. In order to obtain $\overline{C_{L}^{x x}}$, we begin with Eq. (73) in the following form:

$$
\begin{aligned}
J(\lambda, \Gamma \mid y)= & \frac{\tau(0, \Gamma)}{u(0, \Gamma)} \frac{\sinh ^{2}\left(\Delta \Gamma_{I}\right)}{\sinh (\Delta \Gamma)} \Delta \frac{\left(2 \lambda-\delta+\Delta \operatorname{coth}\left(\Delta \Gamma_{I}\right)\right)^{2}}{(2 \delta-\lambda)^{2}} e^{-(2 \lambda+3 \delta) \Gamma+2(\lambda+\delta) \Gamma_{I}} \\
& \times\left[\frac{1}{[(\delta+\lambda) \sin (\Delta \Gamma)+\Delta \cosh (\Delta \Gamma)]^{2}}+\frac{2(2 \delta-\lambda) \sinh (\Delta \Gamma)}{[(2 \lambda-\delta) \sinh (\Delta \Gamma)+\Delta \cosh (\Delta \Gamma)]^{3}}-\frac{1}{[(2 \lambda+\delta) \sinh (\Delta \Gamma)+\Delta \cosh (\Delta \Gamma)]^{2}}\right] .
\end{aligned}
$$

First, we perform an inverse Laplace transform in $y$ to recover the length dependence: 


$$
\begin{aligned}
& J(\lambda, \Gamma \mid L)=\sum_{n=1}^{\infty}(-1)^{n} \frac{\tau(0, \Gamma)}{u(0, \Gamma)} \frac{\left[(2 \lambda-\delta) \Gamma_{I}+1\right]^{2}}{(2 \delta-\lambda)^{2}} e^{-(2 \lambda+3 \delta) \Gamma+2(\lambda+\delta) \Gamma_{I}} e^{-\left[\delta^{2}+\left(\frac{n \pi}{\Gamma}\right)^{2}\right] L}
\end{aligned}
$$

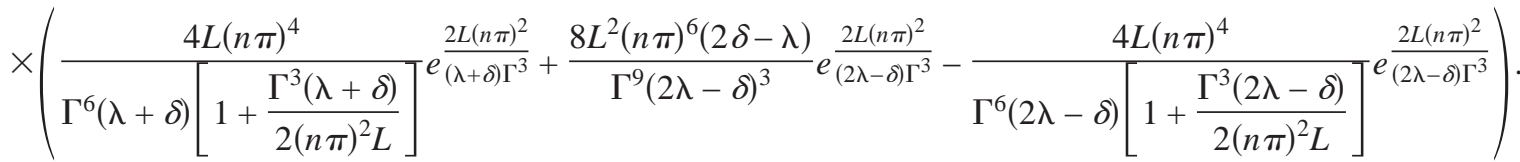

This is obtained by approximating the roots of

$$
a \sinh (\Delta \Gamma)+\Delta \cosh (\Delta \Gamma)
$$

with $a \approx 1$, by $y_{n}=-\delta^{2}-(n \pi / \Gamma)^{2}(1-2 / \Gamma a)$ and expanding Eq. (85) around these roots

$$
a \sinh (\Delta \Gamma)+\Delta \cosh (\Delta \Gamma) \approx \sum_{n=1}^{\infty}(-1)^{n+1} i\left(y-y_{n}\right) \frac{a \Gamma^{2}}{2 n \pi}
$$

The roots in Eq. (86) are given as an expansion in powers of $1 / \Gamma$. Since we are interested in $\Gamma \gg 1$, we are content with only the first two terms; in fact, as can be seen by the following Eq. (87), only the first nonvanishing power of $1 / \Gamma$ contributes to the average correlations. In addition, Eq. (86) is only valid for $y_{n} \ll 1$, i.e., for $n \pi<\Gamma$. But since we are interested in the large length behavior of the correlations, we can restrict our calculation to small values of $n$, as they give the slowest decaying term in the correlations.

The desired result is obtained by performing the $\Gamma$ integral. This integral is dominated by the large exponent in

$$
e^{-\left(\frac{n \pi}{\Gamma}\right)^{2} L-(2 \lambda+\delta) \Gamma\left\{+O[\log (\Gamma)]+O\left(L \Gamma^{-3}\right)\right\}}=e^{g(\Gamma)},
$$

which has a saddle point at $\Gamma_{S}=\left[2(n \pi)^{2} L / 2 \lambda+\delta\right]^{1 / 3}$. The exponential dependence then becomes

$$
e^{g(\Gamma)} \approx e^{g\left(\Gamma_{S}\right)-\frac{1}{2} 3\left[\frac{(2 \lambda+\delta)^{4}}{2(n \pi)^{2} L}\right]^{1 / 3}\left(\Gamma-\Gamma_{S}\right)^{2}}
$$

and the saddle-point integration yields

$$
\begin{aligned}
& J(\lambda, L) \approx \sum_{n=1}^{\infty}(-1)^{n+1} 2^{2 / 3} \sqrt{\frac{\pi}{3}}(n \pi)^{1 / 3} \\
& \times \frac{1}{L^{5 / 6}} e^{-\delta^{2} L-3 L^{1 / 3}(n \pi)^{2 / 3}(\lambda+\delta / 2)^{2 / 3}} \times e^{2 \Gamma_{I}(\lambda+\delta)} \\
& \times\left(\frac{2 \lambda \Gamma_{I}+1}{\lambda-2 \delta}\right)^{2} \frac{1}{(2 \lambda+\delta)^{2 / 3}} \\
& \times\left(e^{2-\frac{\delta}{\lambda+\delta}} \frac{(2 \lambda+\delta)^{3}}{(\lambda+\delta)(3 \lambda+2 \delta)}\right. \\
& \left.+e^{1-\frac{2 \delta}{2 \lambda-\delta}} \frac{(2 \delta-\lambda)(2 \lambda+\delta)^{2}}{(2 \lambda-\delta)^{2}}-e^{2-\frac{\delta}{\lambda+\delta}} \frac{(2 \lambda+\delta)^{3}}{(4 \lambda)(2 \lambda-\delta)}\right) .
\end{aligned}
$$

This result is valid for the critical regime $\left(1 / \delta^{2} \gg L\right)$ when
$1 / L^{1 / 3} \ll \lambda \ll L$, and away from the critical regime $\left(1 / \delta^{2}\right.$ $\ll L$ ), away from $\lambda=0$. To get the equivalent expression for $\lambda \Rightarrow 0$, we need to be more careful with the third term of Eq. (89) and get the next-order corrections. Note that there is no singularity in this expression at $\lambda=2 \delta$; this will have implications for the off-critical large $L$ behavior.

\section{Result}

To get the final result for $\overline{C_{L}^{x x}}$, all that remains is to set $\lambda \Rightarrow 1$. Neglecting terms suppressed by factors of $\delta \ll 1$, we obtain

$$
\overline{C_{L}^{x x}} \approx A_{0}^{x x} \frac{1}{L^{5 / 6}} e^{-\delta^{2} L-3 L^{1 / 3}(\pi)^{2 / 3}(1+\delta / 2)^{2 / 3}},
$$

Also, for the exchange-energy correlations we obtain

$$
\overline{C_{L}^{B B}} \approx A_{0}^{z z} \frac{1}{L^{5 / 6}} e^{-\delta^{2} L-3 L^{1 / 3}(\pi)^{2 / 3}(1-\delta / 2)^{2 / 3}},
$$

where $A_{0}^{x x}$ and $A_{0}^{z z}$ are nonuniversal coefficients. When the chain is not critical, we notice that the exponential decay is controlled by the same correlation length, $\xi=1 / \delta^{2}$, as the order-parameter correlation function.

\section{B. Average cross correlations}

\section{Derivation of the Average}

In complete analogy with the derivation of the previous section, we proceed from Eq. (82) in the following form:

$$
\begin{aligned}
J(\lambda, \Gamma \mid y) \approx & \frac{\left[\left(2 \lambda \Gamma_{I}+1\right)^{2}-\left(\delta \Gamma_{I}\right)^{2}\right]}{\lambda} e^{-2 \lambda\left(\Gamma-\Gamma_{I}\right) \frac{\Delta^{2}}{\sinh (\Delta \Gamma)}} \\
& \times\left(\frac{1}{(\lambda+2 \delta)} \frac{1}{[(\lambda-\delta) \sinh (\Delta \Gamma)+\Delta \cosh (\Delta \Gamma)]}\right. \\
& +\frac{1}{(\lambda-2 \delta)} \frac{1}{[(\lambda+\delta) \sinh (\Delta \Gamma)+\Delta \cosh (\Delta \Gamma)]} \\
& +\frac{(\lambda-\delta)}{\delta(2 \delta-\lambda)} \frac{1}{[(2 \lambda-\delta) \sinh (\Delta \Gamma)+\Delta \cosh (\Delta \Gamma)]} \\
& +\frac{(\lambda+\delta)}{\delta(2 \delta+\lambda)} \frac{1}{[(2 \lambda+\delta) \sinh (\Delta \Gamma)+\Delta \cosh (\Delta \Gamma)]} .
\end{aligned}
$$

Performing an inverse Laplace transform in $y$ and then per- 
forming a saddle-point integration in $\Gamma$, we find:

$$
\begin{aligned}
& J(\lambda \mid L) \approx \sum_{n=1}^{\infty} e^{2 \lambda \Gamma_{I}} \frac{\left(\left(2 \lambda \Gamma_{I}+1\right)^{2}-\left(\delta \Gamma_{I}\right)^{2}\right)}{\lambda^{2 / 3}} 2 \sqrt{\frac{\pi}{3}}(n \pi)^{1 / 3} \frac{1}{L^{5 / 6}} \\
& \times e^{-\delta^{2} L-3 L^{1 / 3}(n \pi)^{2 / 3} \lambda^{2 / 3}}\left(\frac{e^{2+\frac{2 \delta}{\lambda-\delta}}}{\lambda-2 \delta}+\frac{e^{2-\frac{2 \delta}{\lambda+\delta}}}{\lambda+2 \delta}\right. \\
& \left.-\frac{(\lambda-\delta) e^{1+\frac{\delta}{2 \lambda-\delta}}}{\delta(\lambda-2 \delta)}+\frac{(\lambda+\delta) e^{1-\frac{\delta}{2 \lambda+\delta}}}{\delta(\lambda+2 \delta)}\right) \text {. }
\end{aligned}
$$

This result, as well as Eq. (89), is valid for $1 / L^{1 / 3} \ll \lambda \ll L$ for all small or zero $\delta$.

\section{Result}

The average $x-B$ correlation is obtained from the above by setting $\lambda=1$ :

$$
\overline{C_{L}^{x-B}} \approx-A_{0}^{x-B} \frac{1}{L^{5 / 6}} e^{-\delta^{2} L-3 L^{1 / 3}(\pi)^{2 / 3}}
$$

which is almost the same as Eq. (90), but the $\delta$ dependence of the above result is strictly symmetric with respect to $\delta$, as expected for an object that is self-dual.

\section{Typical correlations}

One of the striking features of random quantum systems is that typical correlations are usually very different from average correlations. Average correlation functions can be, as here, dominated by samples (or spatial regions) with anomalously strong correlations. The typical correlations are much smaller and decay faster with distance. Indeed, for the random Ising chain, the typical correlations hold for almost all long-but-finite samples. The average end-to-end correlations are dominated by extremely rare samples.

In the off-critical regime,the typical correlations are well characterized by the average log correlations

$$
C_{\text {typical }}=e^{-\overline{\log (C)}}=e^{-\bar{\Lambda}}
$$

However, at the critical point, $-\overline{\log (C)}=-\bar{\Lambda}$ is of the same order as the logarithm of the typical correlations, but the typical correlations will also have a very wide spread. More precisely, there is a random proportionality constant relating the $\log$ of the correlations to its average; this constant is random and widely varying. We will first investigate the typical correlations at the critical point, and then consider the off-critical regime.

\section{Typical correlations at the critical point}

The average log correlations $\bar{\Lambda}$ are easily found at the critical point. Going back to Eq. (73) and setting $\delta=0$, we see that the Laplace transform in $L$ of the $x-x \log$ correlations is given by

$$
\begin{aligned}
\bar{\Lambda}^{x x}(y)= & -\left.\int_{\Gamma_{I}}^{\infty} \frac{\partial J(\lambda, \Gamma, y)}{\partial \lambda}\right|_{\lambda \Rightarrow 0} d \Gamma \\
= & \int_{\Gamma_{I}}^{\infty} \frac{2 \tanh (\Gamma \sqrt{y})\left[3\left(\Gamma-3 \Gamma_{I}\right) \sqrt{y}+10 \tanh (\Gamma \sqrt{y})\right]}{y \cosh (\Gamma \sqrt{y})^{3}} d \Gamma \\
= & \frac{2}{y^{3 / 2}}\left(3 \int_{\sqrt{y} \Gamma_{I}}^{\infty} \frac{\sinh (x)}{\cosh (x)} x d x+10 \int_{\sqrt{y} \Gamma_{I}} \frac{\sinh ^{2}(x)}{\cosh ^{5}(x)} d x\right. \\
& \left.-9 \sqrt{y} \Gamma_{I} \int_{\sqrt{y} \Gamma_{I}}^{\infty} \frac{\sinh ^{4}(x)}{\cosh ^{4}(x)} d x\right) \approx \frac{7 \pi}{4 y^{3 / 2}}+O\left(\frac{1}{y}\right)
\end{aligned}
$$

By performing the inverse Laplace transform, we get

$$
\bar{\Lambda}_{L}^{x x} \approx \frac{7 \sqrt{\pi}}{4} \sqrt{L}+O(1) \approx 3.1 \sqrt{L} .
$$

The result in Eq. (96) should be compared with the critical behavior of the average correlation:

$$
\log \left(\overline{e^{-\Lambda}}\right) \approx-3 \pi^{2 / 3} L^{1 / 3}
$$

The typical correlations decay as $\sim e^{-k \sqrt{L}}$, with $k$ of order unity but random with a computable universal distribution. But the average correlation function is $\sim e^{-c^{\prime} L^{1 / 3}}$, which decays much more slowly. As claimed above, this means that realizations of the quenched randomness that have an exponentially low probability dominate the average.

By differentiating Eq. (95) once more with respect to $\lambda$, we get $\overline{\left(\Lambda_{(y)}^{x x}\right)^{2}}$. Using that we get for the standard deviation of $\Lambda^{x x}$ :

$$
\sqrt{\overline{\left(\Lambda^{x x}\right)^{2}-\left(\overline{\Lambda^{x x}}\right)^{2}}} \approx 5.6 \sqrt{L} \text {. }
$$

The distribution of $\Lambda / \sqrt{L}$ is thus indeed nontrivial for long critical chains.

For the cross-correlation function, $J \hat{\sigma}^{z^{\prime}} \hat{\sigma}^{z}-h \hat{\sigma}^{x}$, the result we get for the average log correlation is (by a similar calculation)

$$
\begin{gathered}
\overline{\Lambda_{L}^{x-B}} \approx \frac{16}{3 \sqrt{\pi} \sqrt{L}+O(1)} \approx 3.0 \sqrt{L}, \\
\sqrt{\overline{\left(\Lambda^{x-B}\right)^{2}-\left(\overline{\Lambda^{x-B}}\right)^{2}}} \approx 5.4 \sqrt{L} .
\end{gathered}
$$

Note the similar-but not identical—behavior of the two results (99) and (96).

\section{Off-critical $x$-x correlations}

To investigate the $x$ - $x$ energy correlations in the offcritical regime, we pursue a different course of action. Instead of setting $\lambda$ to 1 , we invert the Laplace transform with respect to $\lambda$ in expression (73) and obtain $J(\Lambda, \Gamma \mid y)$ in terms of $\Lambda$. 
Equation (73) can be written in the following form:

$$
\begin{aligned}
J(\lambda, \Gamma \mid y)= & \frac{\tau(0, \Gamma)}{u(0, \Gamma) \frac{\sinh ^{2}\left(\Delta \Gamma_{I}\right)}{\sinh (\Delta \Gamma)^{3}} \Delta[2 \lambda-\delta} \\
& \left.+\Delta \operatorname{coth}\left(\Delta \Gamma_{I}\right)\right]^{2} e^{-2 \lambda\left(\Gamma-\Gamma_{I}\right)} e^{+2 \delta \Gamma_{I}-3 \delta \Gamma} \\
& \times\left[\frac{1}{(3 \delta+\Delta \operatorname{coth}(\Delta \Gamma))^{2}(\delta+\lambda+\Delta \operatorname{coth}(\Delta \Gamma))^{2}}\right. \\
& +\frac{2}{[3 \delta+\Delta \operatorname{coth}(\Delta \Gamma)]^{3}[\delta+\lambda+\Delta \operatorname{coth}(\Delta \Gamma)]} \\
& +\frac{4}{[3 \delta+\Delta \operatorname{coth}(\Delta \Gamma)][2 \lambda-\delta+\Delta \operatorname{coth}(\Delta \Gamma)]^{3}} \\
& \left.-\frac{4}{[3 \delta+\Delta \operatorname{coth}(\Delta \Gamma)]^{3}[2 \lambda-\delta+\Delta \operatorname{coth}(\Delta \Gamma)]}\right] .
\end{aligned}
$$

This form of $J(\lambda, \Gamma \mid y)$ lends itself to inverting the Laplace transform and recovering the $\Lambda$ dependence. This gives (neglecting $\Gamma_{I}$, as before)

$$
\begin{aligned}
& J(\Lambda, \Gamma \mid y) \approx \frac{\tau(0, \Gamma)}{u(0, \Gamma)} \frac{1}{\sinh (\Delta \Gamma)^{3}} \Delta^{3} e^{+2 \delta \Gamma_{I}-3 \delta \Gamma} \Theta\left[\Lambda-2\left(\Gamma-\Gamma_{I}\right)\right] \\
& \times\left[\left(\frac{\left[\Lambda-2\left(\Gamma-\Gamma_{I}\right)\right]}{[3 \delta+\Delta \operatorname{coth}(\Delta \Gamma)]^{2}}\right.\right. \\
& \left.+\frac{2}{(3 \delta+\Delta \operatorname{coth}(\Delta \Gamma))^{3}}\right) e^{-\left[\Lambda-2\left(\Gamma-\Gamma_{I}\right)\right][\delta+\Delta \operatorname{coth}(\Delta \Gamma)]} \\
& +\left(\frac{2\left[\Lambda-2\left(\Gamma-\Gamma_{I}\right)\right]^{2}}{[3 \delta+\Delta \operatorname{coth}(\Delta \Gamma)]}\right. \\
& \left.\left.-\frac{4}{[3 \delta+\Delta \operatorname{coth}(\Delta \Gamma)]^{3}}\right) e^{-\frac{1}{2}\left[\Lambda-2\left(\Gamma-\Gamma_{I}\right)\right][-\delta+\Delta \operatorname{coth}(\Delta \Gamma)]}\right] \text {, }
\end{aligned}
$$

with $\Theta$ the Heaviside step function. Off critical, for long enough chains, specifically with, $L \gg \xi \approx 1 / \delta^{2}$ and the concomitant log-energy scale $\Gamma \delta \gg 1$, one can expand

$$
\Delta \approx \delta+\frac{y}{2 \delta}
$$

and hence obtain

$$
\begin{aligned}
J(\Lambda, \Gamma \mid y) \approx & |\delta|^{3} e^{+2 \delta \Gamma_{I}-(\delta+3|\delta|) \Gamma} \Theta\left[\Lambda-2\left(\Gamma-\Gamma_{I}\right)\right] \\
& \times\left[\left(\frac{\left[\Lambda-2\left(\Gamma-\Gamma_{I}\right)\right]}{(4 \delta)^{2}}\right.\right. \\
& \left.+\frac{2}{(4 \delta)^{3}}\right) e^{-\left[\Lambda-2\left(\Gamma-\Gamma_{I}\right)\right](\delta+|\delta|)} e^{-\frac{y}{2|\delta|}\left[\Lambda-2\left(\Gamma-\Gamma_{I}\right)\right]} \\
& +\left(\frac{2\left[\Lambda-2\left(\Gamma-\Gamma_{I}\right)\right]^{2}}{(4 \delta)}-\frac{4}{(4 \delta)^{3}}\right) \\
& \left.\times e^{-\frac{1}{2}\left[\Lambda-2\left(\Gamma-\Gamma_{I}\right)\right](-\delta+|\delta|)} e^{-\frac{y}{4|\delta|}\left[\Lambda-2\left(\Gamma-\Gamma_{I}\right)\right]}\right]
\end{aligned}
$$

From the simple form of the $y$ dependence in this limit, one can invert the Laplace transform by inspection to obtain the $L$ dependence.

In the paramagnetic phase $(\delta>0), J(\Lambda, \Gamma \mid L)$ is sharply peaked for long chains at $2\left(\Gamma-\Gamma_{I}\right)+4 \delta L$. In the ferromagnetic phase $(\delta<0)$, it is instead sharply peaked at $2\left(\Gamma-\Gamma_{I}\right)$ $+2|\delta| L$. Integrating over $\Gamma$ gives an exponential decay in $L$. Thus, the distributions of the end-to-end transverse field log correlations of long off-critical samples will have the form

$$
\begin{aligned}
f^{x x}(\Lambda, L) & =\int_{\Gamma_{I}}^{\infty} J(\Lambda, \Gamma \mid L) d \Gamma \\
& \sim\left\{\begin{array}{cc}
e^{-2 \delta(\Lambda-4 \delta L)} \Theta(\Lambda-4 \delta L) & \delta>0 \\
e^{-|\delta|(\Lambda-2|\delta| L)} \Theta(\Lambda-2|\delta| L) & \delta<0
\end{array} .\right.
\end{aligned}
$$

(Recall that $\eta$ is the Heaviside step function.) This behavior, with the exponential decay of almost all samples with a characteristic length that is much shorter than the correlation length, is similar to that of the order-parameter correlations in the paramagnetic phase as discussed in Sec. I C of the introduction.

\section{Off-critical $x-B$ correlations}

The same analysis can be applied to the $x-B$ correlation function. The mathematical expressions are simpler, but the result is more interesting. Since this correlation function is symmetric with respect to $\delta$, we can choose to carry out the analysis in the paramagnetic phase, $\delta>0$. Using the same simplifying limit as before $\left(\Gamma_{I} \Rightarrow 0, \Gamma \delta \gg 1, y \ll \delta^{2}\right)$, and keeping only the dominating terms in the disordered phase, Eq. (82) becomes

$$
J(\lambda, \Gamma \mid y) \approx e^{-2 \lambda\left(\Gamma-\Gamma_{I}\right)-2 \delta \Gamma} \delta^{2}\left(\frac{1 / y}{\lambda-\frac{y}{4 \delta}}-\frac{1 / y}{\lambda-\frac{y}{2 \delta}}\right) .
$$

The inverse Laplace transform in $\lambda$ and $y$ of this leads to

$$
\begin{aligned}
J(\lambda, \Gamma \mid y) \approx & e^{-2 \delta \Gamma} \delta^{2}\left(\Theta\left[\Lambda-2\left(\Gamma-\Gamma_{I}\right)-2 \delta L\right]\right. \\
& \left.-\Theta\left[\Lambda-2\left(\Gamma-\Gamma_{I}\right)-4 \delta L\right]\right) .
\end{aligned}
$$

By integrating over $\Gamma$, we get for the distribution of the endto-end $\log$ cross-correlations, $\Lambda=\Lambda_{L}^{x-B}$, of long off-critical chains,

$$
\begin{aligned}
f_{(\Lambda, L)}^{x-B} & =\int_{\Gamma_{I}}^{\infty} J(\Lambda, \Gamma \mid L) d \Gamma \\
& \sim \begin{cases}0, & \Lambda<2|\delta| L \\
1-e^{-\delta(\Lambda-2|\delta| L)}, & 2|\delta| L<\Lambda<4|\delta| L . \\
\left(1-e^{-2 \delta^{2} L}\right) e^{-|\delta|(\Lambda-4|\delta| L)}, & \Lambda>4|\delta| L\end{cases}
\end{aligned}
$$

Most long samples will have $2 \delta L<\Lambda<4 \delta L$; remembering that this expression is valid only for $L \delta^{2} \gg 1$, we see that the distribution will be roughly constant in this range. For larger $\Lambda$, the distribution decays exponentially.

In the ferromagnetic phase, the same result for the cross correlations will obtain with $\delta$ replaced by $|\delta|$. The behavior 
in this phase contrasts with that of the $x-x$ correlations whose distribution of $\Lambda$ is peaked near $2|\delta| L$, and thus are typically stronger than the cross correlations.

\section{Energy-energy correlations and the energy gap}

Looking at the earlier results for the average of the energy gap, $\Delta E$, of finite chains, ${ }^{6}$ we observe a strong resemblance to the results obtained here for the average $E-E$ correlations. In particular Eq. (60) in Ref. 6,

$$
\overline{\Delta E} \sim L^{1 / 6} e^{-\frac{3}{2}\left(\frac{\pi^{2} L}{2}\right)^{1 / 3}},
$$

gives the average gap at the critical point $\delta=0$. We see that

$$
2 \log \overline{\Delta E} \approx \log \overline{C_{L}^{E E}} .
$$

Some relation between the gap and the energy correlations is to be expected, but the behavior of the two quantities is surprising in its degree of the similarity. We will see that this relationship between the gap and the energy correlations arises in the structure of the RG flow.

In Eq. (103), the Heaviside function $\Theta\left[\Lambda-2\left(\Gamma-\Gamma_{I}\right)\right]$ implies that $-\log \left(C_{(L)}^{\Gamma}\right)>2\left(\Gamma-\Gamma_{I}\right)$. Since the appropriate $\Gamma$ at which the decimation establishing this correlation occurs is $\Gamma=-\log \Delta E$, this implies that

$$
C_{L}^{x x}<\left(\frac{\Delta E}{\Omega_{I}}\right)^{2} .
$$

To understand this inequality, consider the correlation coefficient $e^{-\Lambda}$ associated with an end site, and compare this to the transverse field $\bar{h}$ on the end spin cluster. The strongest correlations will occur if the chain undergoes a series of bond decimations. Looking at Fig. 6, one can see that in this case, the evolution of $e^{-\Lambda}$ and $\tilde{h}$ are exactly the same; in each end-bond decimation they acquire a factor of the $h_{0} / J_{01}$ at that scale. The strongest correlations dominate the average correlations. Therefore, chains in which the energy correlations and the gap are strongly correlated also dominate the average correlations.

\section{CONCLUSIONS}

In this paper we have investigated the various contributions to the end-to-end energy correlations of random quantum Ising chains in the universal regime of long chains near the quantum phase transition. In principal, the main result obtained here, the Laplace transform of the distribution of the logarithm of the correlation functions (73) and (82), can be used to obtain the complete distributions in the scaling limit. We have explicitly computed the average and the typical correlations, as well as some other aspects of the distributions, in various limits. The average correlations are dominated by exponentially (in the chain length) rare samples. Nevertheless, they still decay as $e^{-C L^{1 / 3}}$ at the critical point. This is in contrast to the power law decay of the average order-parameter correlations. The various components of the energy correlations are qualitatively similar, although their distributions differ. The cross correlations between the order- ing operator, $J \sigma^{z} \sigma^{z}$, and disordering transverse field operator, $h \sigma^{x}$, are negative because of their competing effects; they are also dual to each other.

The average correlations in the off-critical regime decay with the same correlation length: $\xi \sim 1 / \delta^{2}$, as the orderparameter correlations. The typical correlations, however, decay much faster, and have the same correlation-length exponent, $\widetilde{\nu}=1$, as the pure system. In Sec. V C 2 it was shown that the distribution of $\hat{\sigma}_{0}^{x} \hat{\sigma}_{L}^{x}$ is strongly peaked near $\exp ($ $-4 \delta L)$ for $\delta>0$ and near $\exp (-2|\delta| L)$ for $\delta<0$, indicating a surprising asymmetry between the two phases. This asymmetry is a result of the difference between the ordering and disordering components of the energy density and their behavior in the corresponding phases. At the critical point, the typical correlations decay as $\sim e^{-K L^{1 / 2}}$, with $K$ a random variable; this is similar to the typical order-parameter correlations.

The behavior of the end-to-end energy correlations turns out to be related to that of the lowest-energy gap. We explain this in terms of the rare realizations of the quenched randomness that dominate: these are such that the gap and the correlation function involve essentially the same product of ratios of $J$ 's to $h$ 's. The connection between the energy correlations and the energy gap suggests that the energy gap could be probed indirectly using the-in some cases more experimentally accessible - spatial correlations. This connection is reminiscent of that which occurs in conventional ordered phases in which the gap and spatial correlation length are related.

Unlike previous RG calculations of properties of the random transverse field Ising model, the energy correlations required the development of a formalism that goes beyond second-order perturbation theory. Performing the RG transformation by unitary transformations proved to be a useful tool that allows one to follow readily the evolution of effective operators. Here, we have focused on end-to-end correlations because these are far simpler to handle analytically: correlations in the bulk of the chain involve effective operators on both sides of the objects of interest and are much harder to deal with. Nevertheless, they could be computed by numerically keeping track of the needed distributions.

For Ising chains, an alternative method for calculating correlation functions is via othe Wigner-Jordan mapping of Eq. (1) to a free-fermion model. Unfortunately, this method does not offer much advantage: even in the resulting freeparticle problem, the primary obstacle is the diagonalization of the random Hamiltonian. This can be done by numerical diagonalization methods, ${ }^{18,21}$ but these are limited to relatively small systems, and can currently be implemented only on chains of an order of several hundred spins. With such short lengths, numerics can be strongly affected by finite-size effects, and extracting universal-nevermind exact-longsize limits is difficult. ${ }^{27}$ An alternative to direct numerical diagonalization is an iterative real space RG approach to the diagonalization: the optimal strategy is analogous to that which we use here.

The unitary transformation RG method developed in this paper can also be used to study correlations in imaginary time. So far, mostly average quantities have been calculated 
(for instance, see Refs. 18, 22, and 23), but progress on distributions should be possible utilizing the procedure described here (Sec. II). More generally, the present RG method should be applicable to other models both in one dimension and in higher dimensions, with the unitary RG procedure enabling one to follow the flows numerically to obtain information about the behavior of operators of interest at both high and low energies.

\section{ACKNOWLEDGMENTS}

This work has been supported in part by the National Science Foundation via DMR-9976621, DMR-9809363, and the MRSEC at Harvard. One of us (G.R.) was supported in part by a Harvard Merit Fellowship.

\section{APPENDIX: EFFECTS OF END OPERATORS}

When calculating the energy correlation functions, we kept terms that were third order or higher in the perturbation expansion. A conccern to the validity of our treatment is that terms that were produced as fourth- or fifth-order terms at an early stage of the RG flow, $\Gamma_{1}$, become more relevant than terms that we kept that are produced at a later stage of the RG flow, $\Gamma_{2}>\Gamma_{1}$. In this appendix we verify that end operator effects that we excluded in the text can not give rise to leading-order contributions to the various computed energy correlations.

In addition, The flow of the operator $\hat{\sigma}_{0}^{x}$ when the end spin is decimated, will include a term $\hat{\sigma}_{0}^{z} \hat{\sigma}_{1}^{z}$. Naively, as this is an order-rather than a disorder-energy operator, it may make the disordered and ordered phases look indistinguishable as far as the transverse spin correlations are concerned. We will show here that this is not the case, and although these extraneous operators do appear, their contribution is, at best, subdominant.

\section{End site decimation}

In order to prove the above claims, we need to investigate the additional operators that arise when decimating ends. Revisiting the process of decimating an end site, we consider the flow of the operator $h_{0} \hat{\sigma}_{0}^{x}$. The following table describes the series of transformation, and the effective operators that contribute to $h_{0} \hat{\sigma}_{0}^{x}$ :

$$
\begin{array}{ccc} 
& \underline{S} & \underline{h_{0} \hat{\sigma}_{0}^{x}} \\
S_{a}: & -\frac{J_{01}}{2 h_{0}} \hat{\sigma}_{0}^{y} \hat{\sigma}_{1}^{z} & -J_{01} \hat{\sigma}_{0}^{z} \hat{\sigma}_{1}^{z} \\
S_{b}: & \frac{J_{01} h_{1}}{2 h_{0}^{2}} \hat{\sigma}_{0}^{z} \hat{\sigma}_{1}^{y} & -\frac{J_{01} h_{1}}{h_{0}} \hat{\sigma}_{0}^{y} \hat{\sigma}_{1}^{y}+\frac{J_{01}^{2} h_{1}}{h_{0}^{2}} \hat{\sigma}_{1}^{x} \\
S_{c}: & -\frac{J_{01} J_{12} h_{1}}{2 h_{0}^{3}} \hat{\sigma}_{0}^{y} \hat{\sigma}_{1}^{x} \hat{\sigma}_{2}^{z} & -\frac{J_{01} J_{12} h_{1}}{h_{0}^{2}} \hat{\sigma}_{0}^{z} \hat{\sigma}_{1}^{x} \hat{\sigma}_{2}^{z} \\
S_{d}: & -\frac{J_{01} J_{12} h_{1} h_{2}}{2 h_{0}^{4}} \hat{\sigma}_{0}^{z} \hat{\sigma}_{1}^{x} \hat{\sigma}_{2}^{y} & \frac{J_{01}^{2} J_{12} h_{1}^{2}}{h_{0}^{4}} \hat{\sigma}_{1}^{z} \hat{\sigma}_{2}^{z}+\frac{J_{01}^{2}}{h_{0}^{4}} J_{12} h_{1} h_{2} \hat{\sigma}_{1}^{y} \hat{\sigma}_{2}^{y} .
\end{array}
$$

The fourth line and the second line contain the terms that are most likely to give a leading contribution to the correlations; these operators have a nonvanishing expectation value in the ground state of the decimated part. Keeping them all we have

$$
h_{0} \hat{\sigma}_{0}^{x} \Rightarrow \frac{J_{01}^{2} h_{1}}{h_{0}^{2}} \hat{\sigma}_{1}^{x}+\frac{J_{01}^{2}}{h_{0}^{4}} J_{12} h_{1}^{2} \hat{\sigma}_{1}^{z} \hat{\sigma}_{2}^{z}+\frac{J_{01}^{2}}{h_{0}^{4}} J_{12} h_{1} h_{2} \hat{\sigma}_{1}^{y} \hat{\sigma}_{2}^{y} .
$$

Another process that we need to consider is a bond decimation close to the end. If we decimate the first bond, $-J_{01} \hat{\sigma}_{0}^{z} \hat{\sigma}_{1}^{z}$, then $h_{0} \hat{\sigma}_{0}^{x}$ becomes

$$
\begin{aligned}
h_{0} \hat{\sigma}_{0}^{x} & \Rightarrow-\frac{h_{0} h_{1}}{J_{01}} \hat{\sigma}_{0}^{y} \hat{\sigma}_{1}^{y}+\frac{h_{0} h_{1}}{J_{01}^{3}} J_{12} h_{2} \hat{\sigma}_{0}^{y} \hat{\sigma}_{1}^{x} \hat{\sigma}_{2}^{y}=h_{(01)} \hat{\sigma}_{(01)}^{x} \\
& +\frac{1}{\Omega^{2}} h_{(01)} h_{2} J_{(01) 2} \hat{\sigma}_{(01)}^{y} \hat{\sigma}_{2}^{y},
\end{aligned}
$$

where the parentheses signify effective spin clusters. Going further away from the end, a decimation of the second site in the chain, $-h_{1} \hat{\sigma}_{1}^{x}$, will give

$$
\begin{aligned}
h_{0} \hat{\sigma}_{0}^{x} & \Rightarrow h_{0} \hat{\sigma}_{0}^{x}+\frac{1}{h_{1}^{2}} \frac{J_{01} J_{12}}{h_{1}} h_{0} h_{2} \hat{\sigma}_{0}^{y} \hat{\sigma}_{1}^{x} \hat{\sigma}_{2}^{y}=h_{0} \hat{\sigma}_{0}^{x} \\
& +\frac{1}{h_{1}^{2}} J_{02} h_{0} h_{2} \hat{\sigma}_{0}^{y} \hat{\sigma}_{2}^{y},
\end{aligned}
$$

with site 1 eliminated. Note that site 0 is still the first site, but site 2 is now the next.

From the above processes, a pattern emerges. The fifthorder perturbation calculation above produces two dangerous operators:

$$
\hat{\sigma}_{0}^{z} \hat{\sigma}_{1}^{z}, \hat{\sigma}_{0}^{y} \hat{\sigma}_{1}^{y} \text {. }
$$

We need to verify that these operators do not produce leading-order correlations. The first step is to observe that instead of seeing the bare operators, $\hat{\sigma}_{0}^{x}, \hat{\sigma}_{0}^{z} \hat{\sigma}_{1}^{z}, \hat{\sigma}_{0}^{y} \hat{\sigma}_{1}^{y}$, appearing with varying prefactors, we see them appearing in the combinations $h_{0} \hat{\sigma}_{0}^{x}$, $\left(1 / \Omega^{2}\right) h_{0}^{2} J_{01} \hat{\sigma}_{0}^{z} \hat{\sigma}_{1}^{z},\left(1 / \Omega^{2}\right) h_{0} J_{01} h_{1} \hat{\sigma}_{0}^{y} \hat{\sigma}_{1}^{y}$, where $\Omega$ is the energy scale at which the operator appeared. There may be additional prefactors, which we will consider shortly, but first let us establish that these forms have an invariant structure.

\section{Invariant Operators}

In this section we will show that the form of the operators defined as follows:

$$
\begin{gathered}
h_{0} \hat{\sigma}_{0}^{x}, \\
h_{0}^{2} J_{01} \hat{\sigma}_{0}^{z} \hat{\sigma}_{1}^{z}, \\
h_{0} J_{01} h_{1} \hat{\sigma}_{0}^{y} \hat{\sigma}_{1}^{y},
\end{gathered}
$$

is preseved during the RG flow. The coefficients $h_{0}, h_{0}^{2} J_{01}$, and $h_{0} J_{01} h_{1}$ will only be replaced by their renormalized counterparts every time that a decimation affects them. This is demonstrated in the following examples.

The simplest example is the operator $h_{0} \hat{\sigma}_{0}^{x}$. Under the decimation of $-J_{01} \hat{\sigma}_{0}^{z} \hat{\sigma}_{1}^{z}$, 


$$
h_{0} \hat{\sigma}_{0}^{x} \Rightarrow-\frac{h_{0} h_{1}}{J_{01}} \hat{\sigma}_{0}^{y} \hat{\sigma}_{1}^{y}=h_{(01)}^{\mathrm{eff}} \hat{\sigma}_{(01)}^{x} .
$$

Another example is the operator $\left(1 / \Omega^{2}\right) h_{0}^{2} J_{01} \hat{\sigma}_{0}^{z} \hat{\sigma}_{1}^{z}$. The decimation of the second bond, $-J_{12} \hat{\sigma}_{1}^{z} \hat{\sigma}_{2}^{z}$, modifies this operator as follows:

$$
h_{0}^{2} J_{01} \hat{\sigma}_{0}^{z} \hat{\sigma}_{1}^{z} \Rightarrow h_{0}^{2} J_{0(12)} \hat{\sigma}_{0}^{z} \hat{\sigma}_{(12)}^{z}
$$

In the case of the operator $\left(1 / \Omega^{2}\right) h_{0} J_{01} h_{1} \hat{\sigma}_{0}^{y} \hat{\sigma}_{1}^{y}$, the corresponding transformation $S_{a}=\left(h_{2} / 2 J_{12}\right) \hat{\sigma}_{1}^{z} \hat{\sigma}_{2}^{y}$ yields

$$
h_{0} J_{01} h_{1} \hat{\sigma}_{0}^{y} \hat{\sigma}_{1}^{y} \Rightarrow h_{0} J_{0(12)} \frac{h_{1} h_{2}}{J_{12}} \hat{\sigma}_{0}^{y} \hat{\sigma}_{1}^{x} \hat{\sigma}_{2}^{y}=h_{0} J_{0(12)} h_{(12)} \hat{\sigma}_{0}^{y} \hat{\sigma}_{(12)}^{y} \text {. }
$$

Decimating the second site in the chain, $-h_{1} \hat{\sigma}_{1}^{x}$ involves the transformation $S_{a}=\left(J_{12} / h_{1}\right) \hat{\sigma}_{1}^{y} \hat{\sigma}_{2}^{z}$, yielding the following flow for the two operators $\left(1 / \Omega^{2}\right) h_{0}^{2} J_{01} \hat{\sigma}_{0}^{z} \hat{\sigma}_{1}^{z}$, $\left(1 / \Omega^{2}\right) h_{0} J_{01} h_{1} \hat{\sigma}_{0}^{y} \hat{\sigma}_{1}^{y}$ :

$$
h_{0}^{2} J_{01} \hat{\sigma}_{0}^{z} \hat{\sigma}_{1}^{z} \Rightarrow h_{0}^{2} \frac{J_{01} J_{12}}{h_{1}} \hat{\sigma}_{0}^{z} \hat{\sigma}_{1}^{x} \hat{\sigma}_{2}^{z}=h_{0}^{2} J_{02}^{\mathrm{eff}} \hat{\sigma}_{0}^{z} \hat{\sigma}_{2}^{z}
$$

The second transformation in this same decimation process is $S_{b}=\left(J_{12} h_{2} / h_{1}^{2}\right) \hat{\sigma}_{1}^{z} \hat{\sigma}_{2}^{y}$, which gives rise to:

$$
h_{0} J_{01} h_{1} \hat{\sigma}_{0}^{y} \hat{\sigma}_{1}^{y} \Rightarrow h_{0} \frac{J_{01} J_{12}}{h_{1}} h_{1} \frac{h_{2}}{h_{1}} \hat{\sigma}_{0}^{y} \hat{\sigma}_{1}^{x} \hat{\sigma}_{2}^{y}=h_{0} J_{02}^{\mathrm{eff}} h_{2} \hat{\sigma}_{0}^{y} \hat{\sigma}_{2}^{y}
$$

In all cases, the flow due to decimations leaves the three forms of the end operators invariant. We excluded here the cases of a decimation of the first site or bonds; these are considered below.

\section{Displacement prefactors}

As mentioned before and seen from the results of Eq. (A1), there are still multiplicative prefactors coming before the invariant operator forms. In Eq. (A2), for instance, all three operators from Eq. (A6) have the prefactor $\left(J_{01} / h_{0}\right)^{2}$. This suppression can be associated with the displacement of the edge to the next undecimated site. With the help of Eq. (A1), it can be easily shown that an end site $\left(-h_{0} \hat{\sigma}_{0}^{x}\right)$ decimation leads to the following flows:

$$
\begin{gathered}
h_{0} \hat{\sigma}_{0}^{x} \Rightarrow\left(\frac{J_{01}}{h_{0}}\right)^{2} h_{(01)} \hat{\sigma}_{(01)}^{x}, \\
h_{0}^{2} J_{01} \hat{\sigma}_{0}^{z} \hat{\sigma}_{1}^{z} \Rightarrow\left(\frac{J_{01}}{h_{0}}\right)^{2} h_{1} J_{12} h_{2} \hat{\sigma}_{1}^{y} \hat{\sigma}_{2}^{y}, \\
h_{0} J_{01} h_{1} \hat{\sigma}_{0}^{y} \hat{\sigma}_{1}^{y} \Rightarrow\left(\frac{J_{01}}{h_{0}}\right)^{2} h_{1}^{2} J_{01} \hat{\sigma}_{1}^{z} \hat{\sigma}_{2}^{z} .
\end{gathered}
$$

From the above equation we see that there is a factor $\left(J_{01} / h_{0}\right)^{2}$ associated with the displacement into the chain of the $\hat{\sigma}_{0}^{z} \hat{\sigma}_{1}^{z}, \hat{\sigma}_{0}^{y} \hat{\sigma}_{1}^{y}$ operators. This is repeated partially in the case of a bond decimation of the $-J_{01} \hat{\sigma}_{0}^{z} \hat{\sigma}_{1}^{z}$ :

$$
\begin{gathered}
h_{0}^{2} J_{01} \hat{\sigma}_{0}^{z} \hat{\sigma}_{1}^{z} \Rightarrow h_{(01)}^{2} J_{(01) 2} \hat{\sigma}_{(01)}^{z} \hat{\sigma}_{2}^{z} \\
h_{0} J_{01} h_{1} \hat{\sigma}_{0}^{y} \hat{\sigma}_{1}^{y} \Rightarrow\left(\frac{h_{0} h_{1}}{J_{01}^{2}}\right) h_{(01)} J_{(01) 2} h_{2} \hat{\sigma}_{(01)}^{y} \hat{\sigma}_{2}^{y} .
\end{gathered}
$$

\section{Leading-order correlations}

Now that we know how the end operators neglected in the text evolve, and discovered that their forms are invariant, we can show that these operators do not change the leadingorder contributions to the correlation functions of interest. The $x x$ correlation function is

$$
\begin{aligned}
C_{L}^{x x} & =\left\langle h_{0} \hat{\sigma}_{0}^{x} h_{L} \hat{\sigma}_{L}^{x}\right\rangle-\left\langle h_{0} \hat{\sigma}_{0}^{x}\right\rangle\left\langle h_{L} \hat{\sigma}_{L}^{x}\right\rangle \\
& =\left\langle G\left|\left(h_{0} \hat{\sigma}_{0}^{x}-\left\langle h_{0} \hat{\sigma}_{0}^{x}\right\rangle\right)\left(h_{L} \hat{\sigma}_{L}^{x}-\left\langle h_{L} \hat{\sigma}_{L}^{x}\right\rangle\right)\right| G\right\rangle \\
& =\sum_{\psi \neq G}\left\langle G\left|h_{0} \hat{\sigma}_{0}^{x}\right| \psi\right\rangle\left\langle\psi\left|h_{L} \hat{\sigma}_{L}^{x}\right| G\right\rangle,
\end{aligned}
$$

with the sum over all excited states, $\psi$.

As the RG process progresses, all the end operators will be generated several times. However, we need only concern ourselves with the last set of these generated. Previously generated edge operators will have a larger suppression due to the more times they underwent edge displacement.

Considering the last decimation step, which is needed to obtain the $x-x$ correlations and changing the notation so that the remaining effective sites are $\ell, r$ as in the text, we have

$$
\begin{aligned}
C_{L}^{x x} \approx & \frac{e^{-\Lambda_{\ell}-\Lambda_{r}}}{h_{\ell} h_{r}} \sum_{\psi \neq G}\langle H| h_{\ell} \hat{\sigma}_{\ell}^{x}+\frac{1}{\Omega_{\ell}^{2}} h_{\ell}^{2} J_{\ell r} \hat{\sigma}_{\ell}^{z} \hat{\sigma}_{r}^{z} \\
& +\frac{1}{\Omega_{\ell}^{2}} h_{\ell} J_{\ell r} h_{r} \hat{\sigma}_{\ell}^{y} \hat{\sigma}_{r}^{y}|\psi\rangle\langle\psi| h_{r} \hat{\sigma}_{r}^{x}+\frac{1}{\Omega_{r}^{2}} h_{r}^{2} J_{\ell r} \hat{\sigma}_{\ell}^{z} \hat{\sigma}_{r}^{z} \\
& +\frac{1}{\Omega_{r}^{2}} h_{\ell} J_{\ell r} h_{r} \hat{\sigma}_{\ell}^{y} \hat{\sigma}_{r}^{y}|H\rangle .
\end{aligned}
$$

The remaining low-energy parts of the Hamiltonian are

$$
\mathcal{H}=-h_{\ell} \hat{\sigma}_{\ell}^{x}-J_{\ell r} \hat{\sigma}_{\ell}^{z} \hat{\sigma}_{r}^{z}-h_{r} \hat{\sigma}_{r}^{x}
$$

Two cases need to be considered: a site decimation and a bond decimation. In the case of a bond decimation, the ground state of the system is

$$
\left|H^{(L)}\right\rangle=\frac{1}{\sqrt{2}}\left(\left|\downarrow_{\ell}\right\rangle\left|\downarrow_{r}\right\rangle+\left|\uparrow_{\ell}\right\rangle\left|\uparrow_{r}\right\rangle\right)
$$

and we immediately see that the only excitation that contributes in the sum (A15) is the $\hat{\sigma}_{\ell}^{x} \hat{\sigma}_{r}^{x}$ term

$$
C_{L}^{x x} \approx e^{-\Lambda_{\ell}-\Lambda_{r}} h_{\ell} h_{r}
$$

which shows that in this case the dangerous operators [Eq. (A5)] do not contribute to the correlations.

The second case involves a site decimation. Let us assume that the dominant piece in Eq. (A16) is $-h_{\ell} \hat{\sigma}_{\ell}^{x}$ : 


$$
\left|H^{(L)}\right\rangle=\frac{1}{2}\left(\left|\downarrow_{\ell}\right\rangle+\left|\uparrow_{\ell}\right\rangle\right)\left(\left|\downarrow_{r}\right\rangle+\left|\uparrow_{r}\right\rangle\right)
$$

In this case, the contribution of the $\hat{\sigma}_{\ell}^{x} \hat{\sigma}_{r}^{x}$ product is only second order, and we need to consider the unitary transformation that induces this decimation. To lowest order, this is $S_{a}=-\left(J_{\ell r} / 2 h_{\ell}\right) \hat{\sigma}_{\ell}^{y} \hat{\sigma}_{r}^{z}$. Applying this to Eq. (A15), we get

$$
\begin{aligned}
C_{L}^{x x} e^{\Lambda_{\ell}+\Lambda_{r}} h_{\ell} h_{r} \approx & \sum_{\psi \neq G}\langle H| h_{\ell} e^{i S_{a}} \hat{\sigma}_{\ell}^{x} e^{-i S_{a}}+\frac{1}{\Omega_{\ell}^{2}} h_{\ell}^{2} J_{\ell r} \hat{\sigma}_{\ell}^{z} \hat{\sigma}_{r}^{z} \\
& +\frac{1}{\Omega_{\ell}^{2}} h_{\ell} J_{\ell r} h_{r} \hat{\sigma}_{\ell}^{y} \hat{\sigma}_{r}^{y}|\psi\rangle\langle\psi| h_{r} e^{i S_{a}} \hat{\sigma}_{r}^{x} e^{-i S_{a}} \\
& +\frac{1}{\Omega_{r}^{2}} h_{r}^{2} J_{\ell r} \hat{\sigma}_{\ell}^{z} \hat{\sigma}_{r}^{z}+\frac{1}{\Omega_{r}^{2}} h_{\ell} J_{\ell r} h_{r} \hat{\sigma}_{\ell}^{y} \hat{\sigma}_{r}^{y}|H\rangle \\
\approx & \sum_{\psi \neq G}<H\langle H|-h_{\ell} \frac{J_{\ell r}}{h_{\ell}} \hat{\sigma}_{\ell}^{z} \hat{\sigma}_{r}^{z}+\frac{1}{\Omega_{\ell}^{2}} h_{\ell}^{2} J_{\ell r} \hat{\sigma}_{\ell}^{z} \hat{\sigma}_{r}^{z}
\end{aligned}
$$

$$
\begin{aligned}
& +\frac{1}{\Omega_{\ell}^{2}} h_{\ell} J_{\ell r} h_{r} \hat{\sigma}_{\ell}^{y} \hat{\sigma}_{r}^{y}|\psi\rangle\langle\psi| h_{r} \frac{J_{\ell r}}{h_{\ell}} \hat{\sigma}_{\ell}^{y} \hat{\sigma}_{r}^{y} \\
& +\frac{1}{\Omega_{r}^{2}} h_{r}^{2} J_{\ell r} \hat{\sigma}_{\ell}^{z} \hat{\sigma}_{r}^{z}+\frac{1}{\Omega_{r}^{2}} h_{\ell} J_{\ell r} h_{r} \hat{\sigma}_{\ell}^{y} \hat{\sigma}_{r}^{y}|H\rangle \\
= & h_{\ell} h_{r} \frac{J_{\ell r}}{h_{\ell}^{2}}+\frac{\left(h_{\ell}-h_{r}\right) h_{r} J_{\ell r}^{2}}{\Omega_{r}^{2}}-\frac{\left(h_{\ell}-h_{r}\right) h_{r} J_{\ell r}^{2}}{\Omega_{\ell}^{2}} \\
\approx & h_{\ell} h_{r} \frac{J_{\ell r}}{h_{r}^{2}},
\end{aligned}
$$

which is the same result as was derived in the text, while ignoring the additional edge operators from Eq. (A5). In the above we used $h_{\ell}^{2} / \Omega_{r, \ell} \ll 1$.

This demonstration can be repeated for the $x x-z$ correlations and also carried to higher order with the same conclusions. We have thus verified that the energy correlations can be obtained from the leading contributions to the flow of the edge energy operators in each step of the RG.
${ }^{1}$ S. Sachdev, Quantum Phase Transitions (Cambridge University Press, Cambridge, 1999).

${ }^{2}$ D. S. Fisher, Phys. Rev. B 50, 3799 (1994).

${ }^{3}$ D. S. Fisher, Phys. Rev. B 51, 6411 (1995).

${ }^{4}$ B. M. McCoy and T. T. Wu, Phys. Rev. 176, 631 (1968).

${ }^{5}$ B. M. McCoy and T. T. Wu, Phys. Rev. 188, 982 (1969).

${ }^{6}$ D. S. Fisher and A. P. Young, Phys. Rev. B 58, 9131 (1998).

${ }^{7}$ T. Senthil and S. Sachdev, Phys. Rev. Lett. 77, 5292 (1996).

${ }^{8}$ O. Motrunich, S.-C. Mau, D. A. Huse, and D. S. Fisher, Phys. Rev. B 61, 1160 (2000).

${ }^{9}$ C. Pich, A. P. Young, N. K. H. Rieger, and N. Kawashima, Phys. Rev. Lett. 81, 5916 (1998).

${ }^{10}$ A. H. Castrol Neto and B. A. Jones, Phys. Rev. B 62, 14975 (2000).

${ }^{11}$ C. Doty and D. S. Fisher, Phys. Rev. B 45, 2167 (1992).

${ }^{12}$ B. Derrida and H. Hilhorst, J. Phys. C 14, L539 (1981).

${ }^{13}$ A. Aharony and A. B. Harris, Phys. Rev. Lett. 77, 3700 (1996).

${ }^{14}$ S. K. Ma, C. Dasgupta, and C. K. Hu, Phys. Rev. Lett. 43, 1434 (1979).

${ }^{15}$ C. Dasgupta and S. K. Ma, Phys. Rev. B 22, 1305 (1980).

${ }^{16}$ D. S. Fisher, Physica A 263, 222 (1999).

${ }^{17}$ R. Shankar and G. Murthy, Phys. Rev. B 36, 536 (1987).

${ }^{18}$ F. Iglói, R. Juhász, and H. Rieger, Phys. Rev. B 59, 11308 (1999).

${ }^{19}$ F. Wegner, Ann. Phys. (Leipzig) 3, 77 (1994).
${ }^{20}$ S. Kehrein, Nucl. Phys. B 592, 512 (2001).

${ }^{21}$ F. Iglói and H. Rieger, Phys. Rev. B 57, 11404 (1998).

${ }^{22}$ K. Damle, O. Motrunich, and D. A. Huse, Phys. Rev. Lett. 84, 3434 (2000).

${ }^{23}$ O. Motrunich, K. Damle, and D. A. Huse, Phys. Rev. B 63, 134424 (2001).

${ }^{24}$ A. Crisanti and H. Rieger, J. Stat. Phys. 77, 1087 (1994).

${ }^{25}$ By well-behaved distributions, we mean coupling distributions $\rho(J)$ whose behavior near $J=0$ is less divergent than $1 / J \log ^{n}(J)$.

${ }^{26}$ The real space RG scheme presented here can also be applied to simple initial distributions such as bimodal distributions. For example, consider a chain in which the transverse fields are all $h_{1}$, and the Ising energies have value $J_{1}<h_{1}$ with probability $p_{1}$ and value $J_{2}>h_{1}$ with probability $p_{2}=1-p_{1}$. The first step in the $\mathrm{RG}$ prescribes that all bonds with strength $J_{2}$ are decimated to form clusters; this step alone produces a wide variation for the transverse fields. The number of sites in the clusters, $l$, has probability distribution $P(l)=p_{2}^{l-1} p_{1}$. In each cluster, the effective transverse field is $h(l)=h_{1}\left(h_{1} / J_{2}\right)^{l-1}$. Therefore, the distribution of the transverse fields in the renormalized chain at small fields becomes $\rho(h) \sim 1 / h^{1-\log p_{2} / \log \left(h_{1} / J_{2}\right)}$. The process can now continue with decimation of all the sites with transverse field $h_{1}$, and so forth.

${ }^{27}$ An example of such a spurious effect is the numerical evaluation of the dynamical scaling exponent as $z \approx 1.55$ in Ref. 24, whereas the exact result is $z=\infty$. $^{3}$ 